\title{
1 The human ribosomal RNA gene is composed of highly homogenized tandem clusters
}

3 Running title: The human rDNA is quite regular

5 Yutaro Hori ${ }^{1}$, Akira Shimamoto ${ }^{2}$, Takehiko Kobayashi ${ }^{1,3}$

6

71 Institute for Quantitative Biosciences, the University of Tokyo, Tokyo, Japan

82 Faculty of Pharmaceutical Sciences, Sanyo-Onoda City University, Sanyo Onoda, Yamaguchi, Japan

93 Corresponding to tako2015 @iqb.u-tokyo.ac.jp

10

11 Key words: ribosomal RNA gene (rDNA), rDNA copy number, DNA methylation, senescence, genome

12 instability, human, mutation rate, Oxford Nanopore sequencer, gene conversion, homogenization, progeroid 13 syndrome 


\section{ABSTRACT}

16 The structure of the human ribosomal RNA gene clustering region (rDNA) has traditionally been hard to

17 analyze due to its highly repetitive nature. However, the recent development of long-read sequencing technology, such as Oxford Nanopore sequencing, has enabled us to approach the large-scale structure of the genome. Using this technology, we found that human cells have a quite regular rDNA structure. Although each human rDNA copy has some variations in its non-coding region, contiguous copies of rDNA are

21 similar, suggesting that homogenization through gene conversion frequently occurs between copies. Analysis of rDNA methylation by Nanopore sequencing further showed that all of the non-coding regions are heavily methylated, whereas about half of the coding regions are clearly unmethylated. The ratio of unmethylated copies, which are speculated to be transcriptionally active, was lower in individuals with a higher rDNA copy number, suggesting that there is a mechanism that keeps the active copy number stable. Lastly, the rDNA in progeroid syndrome patient cells with reduced DNA repair activity had more unstable copies as compared with control normal cells, although the rate was much lower than previously reported using a Fiber FISH method. Collectively, our results alter the view of rDNA stability and transcription regulation in human cells, indicating the presence of mechanisms for both homogenization to ensure sequence quality and maintenance of active copies for cellular functions. 


\section{INTRODUCTION}

The ribosomal RNA gene (rDNA) is the most abundant repetitive gene in eukaryotic cells. In the budding yeast Saccharomyces cerevisiae, the structure and function of rDNA have been well studied, establishing rDNA as a unique region in the genome. Each unit $(9.2 \mathrm{~kb})$ of rDNA includes two coding regions, 35S precursor ribosomal RNA (rRNA) and 5S rRNA genes, and two non-coding intergenic spacer regions (IGSs) between the genes. The units tandemly repeat ( $\sim 150$ times) in chromosome XII (Petes 1979)(Kobyashi et al., 1998). A unique feature of the yeast rDNA is that it has a system to maintain the quality and quantity of the sequence in order to fulfill the huge demand of ribosomes in the cell (Gangloff et al. 1996) (for review see Kobayashi 2011). The rDNA tends to lose copies through recombination between them because of their repetitive nature and highly activated transcription. To maintain quantity, therefore, the rDNA amplifies more copies when the number is reduced (Kobayashi et al., 1998). As a result, the rDNA is continually undergoing contraction and expansion, and thus is one of the most unstable regions in the genome (Kobayashi 2014).

A DNA binding protein, Fob1 is a key player in the amplification reaction (Kobayashi 2003). It induces recombination for amplification by inhibiting replication at the replication fork barrier (RFB) (Supplemental Fig. S1). The inhibition induces a DNA double strand break at a relatively high frequency, and the repair process increases the number of copies by unequal sister chromatid recombination (Weitao et al. 2003) (Burkhalter and Sogo 2004) (Kobayashi et al. 2004). Recombination is also regulated by noncoding transcription (E-pro transcription) through cohesion dissociation (Kobayashi and Ganley 2005). In terms of quality control of rDNA, the sequences are always homogenized; that is, a copy with mutation is excluded by a Fob1-dependent recombination mechanism, such as gene conversion and contraction of the copies (Ganley and Kobayashi 2007). In fact, the rDNA sequences in the budding yeast are known to be relatively uniform even though about half of the copies are not transcribed (Ganley and Kobayashi 2011). Therefore, we speculate that active recombination in the rDNA maintains the integrity that ensures intact rRNA and the ribosome (Kobayashi 2014).

There is another face of such unstable rDNA - namely, it induces cellular senescence in budding yeast (Ganley and Kobayashi 2014). For example, in the fobl mutant, the rDNA is stable with less recombination and the mutant's lifespan is extended by $\sim 60 \%$ (Takeuchi et al. 2003; Defossez et al. 1999). In 
changes, lifespan is shortened by $\sim 50 \%$ (Saka et al. 2013; Kaeberlein et al. 1999). Because the rDNA is a large unstable region in the genome, its instability may affect the stability of the whole genome and thereby influence lifespan (i.e., the rDNA theory of aging) (Kobayashi 2008).

While we have good knowledge about yeast rDNA and its extra coding functions for aging, there is limited information on human rDNA. One reason is that the human rDNA unit ( $43 \mathrm{~kb})$ is much larger than the yeast rDNA unit $(\sim 9.2 \mathrm{~kb})$ and it includes many small repetitive sequences in the non-coding region. Although the Human Genome Project declared its completion in 2003, it was difficult to assemble the rDNA into its actual composition using the relatively short "reads" that were obtained from the sequencing technology of those days. However, the recent development of DNA polymerase-independent long read sequencing technologies, such as the Oxford Nanopore or PacBio systems, has made it possible to assemble complete sequences of the unexplored regions (Miga et al. 2020).

The human rDNA is comprised of 100 500 copies in a cell (Parks et al. 2018; Agrawal and Ganley 2018). Each unit of rDNA consists of the 45S precursor RNA gene (45S rDNA), whose transcript is processed into mature $18 \mathrm{~S}, 5.8 \mathrm{~S}$, and $28 \mathrm{~S}$ RNAs, and the IGS, which is filled with-small repetitive sequences such as microsatellites and transposons (Fig. 1A). In the IGS, there are two typical repeats: the R repeat and Butterfly/Long repeat. The $\mathrm{R}$ repeat ( $\sim 680 \mathrm{bp}$, typically three copies) is located in the termination region of the 45S rRNA gene. It contains the Sal box that is associated with the transcription factor TTFI (Grummt et al. 1986). TTFI, similar to yeast Fob1, functions to inhibit the replication fork to avoid the collision of RNA and DNA polymerase (Akamatsu and Kobayashi 2015). The Butterfly/Long repeat ( 4,500 bp, typically two copies), which is composed of a Long repeat, CT microsatellite and Butterfly repeat, is located at approximately the center of the IGS (Fig. 1A) (Gonzalez and Sylvester 2001; Agrawal and Ganley 2018). A previous study of rDNA composition in human cells by an in situ hybridization method (Fiber FISH) reported that many irregular units, such as palindromic inverted and incomplete units, account for $\sim 35 \%$ of total copies in rDNA (Caburet et al. 2005). This high rate indicates that there is no effective human rDNA is also related to senescence. Because most progeroid syndromes, such as Werner syndrome and Bloom syndrome, are caused by mutations of the DNA repair machinery, it is plausible that the recombination system to maintain rDNA homogeneity in human cells. In addition, the ratio of these noncanonical rDNA units was found to be increased in cells from progeroid syndrome patients, suggesting that symptoms of these syndromes are caused by instability in the rDNA, which is thought to be one of the most 
unstable DNA regions in human, as in budding yeast (Carrero et al. 2016). Indeed, previous studies have suggested that rDNA copy number varies greatly in cells from Bloom syndrome patients, and palindromic structures have been observed in Werner syndrome cells (Schawalder et al. 2003; Killen et al. 2009). However, it is still unclear whether rDNA instability is an important factor in senescence in human. In terms of the relationship between rDNA and senescence in mammals, rDNA is also known to become methylated during the passage of life (Wang and Lemos 2019). The ratio of rDNA methylation works as an "clock" that tells the individual's age.

To reveal the detailed structure and integrity in the human rDNA cluster at the DNA sequence level, here we developed a method to analyze rDNA-derived long reads obtained by an Oxford Nanopore sequencer. To our surprise, we found that the rDNA array is much more regular than we expected and the sequence similarity between adjacent copies is very high. These results suggest that recombination for homogenization takes place in the human rDNA as it does in the budding yeast.

\section{RESULTS}

\section{Long sequence reads reveal variations among rDNA copies}

To determine the human rDNA structure, we analyzed both publicly available Oxford Nanopore whole genome sequencing (WGS) data from the Human Pangenomics Project (HPGP, https://github.com/humanpangenomics/), and our in-house Cas9-enriched rDNA reads from Epstein-Barr Virus (EBV) transformed B cells (Shafin et al. 2020) and primary fibroblast cells (Supplemental Table S1). Taking advantage of the high copy number of rDNA per cell, we modified the Cas9-enrichment strategy and established a protocol to construct a library at lower cost (Gilpatrick et al. 2020). In short, to enrich the rDNA fragments, we designed four guide RNAs around the 9,500-9,900-nt region from the start site of the coding region (45S rDNA) of the reference sequence; all four sequences were strictly conserved in human and mouse. We designed gRNAs in the coding region because this region is thought to have fewer mutations and the relationship between two neighboring 45S rDNAs can be analyzed in a single read. Total DNA was dephosphorylated with CIP to avoid ligation to the sequencing adapter. The DNA was then digested by Cas9 ribonucleoprotein (RNP).

Because only the Cas9-digested DNA fragments have a phosphorylated-5' end, the sequencing adapters are specifically ligated to the fragments. In analyzing the HPGP genomic data, we removed reads shorter than $40,000 \mathrm{nt}$ to eliminate those that were thought to come from rDNA-derived pseudo genes in non-rDNA 
A
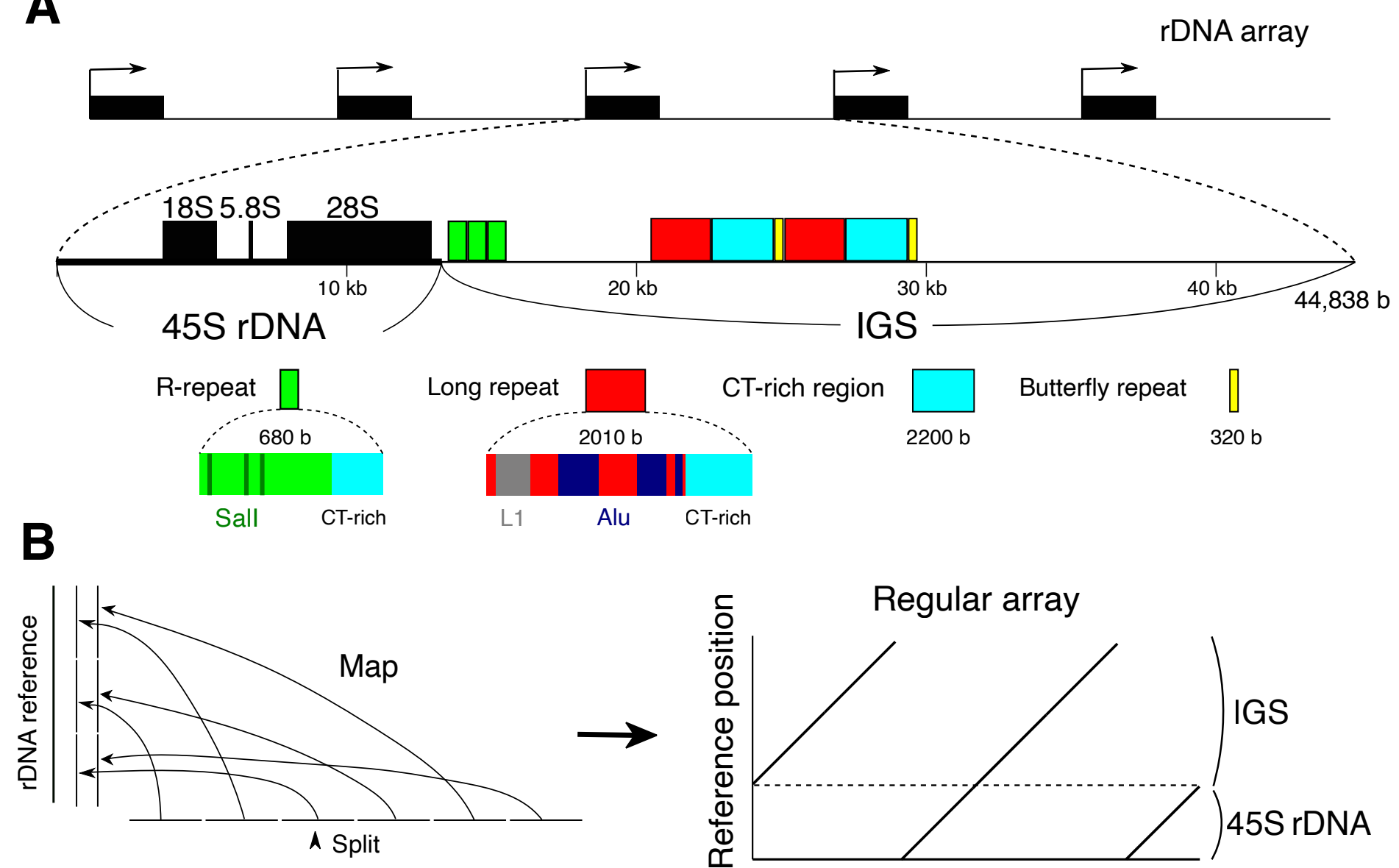

Oxford Nanopore read

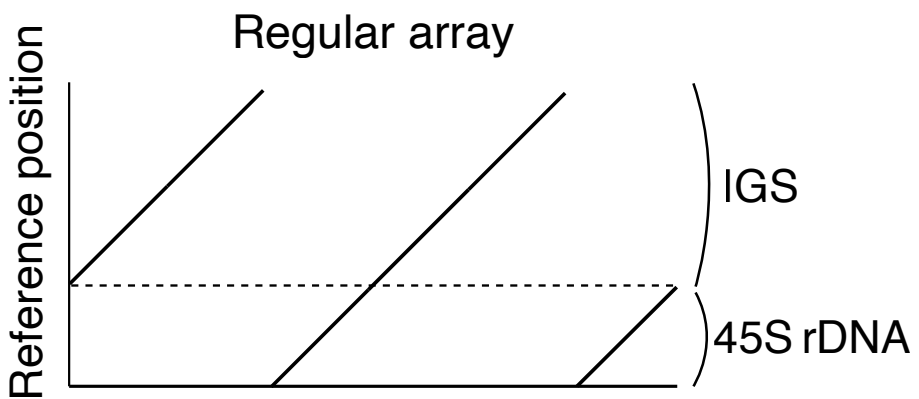

Position in the read

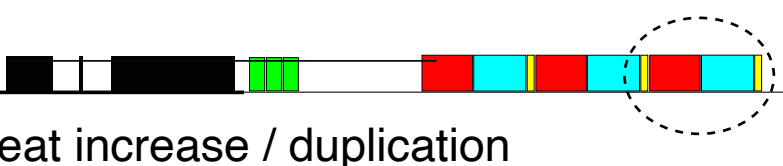

Repeat increase / duplication
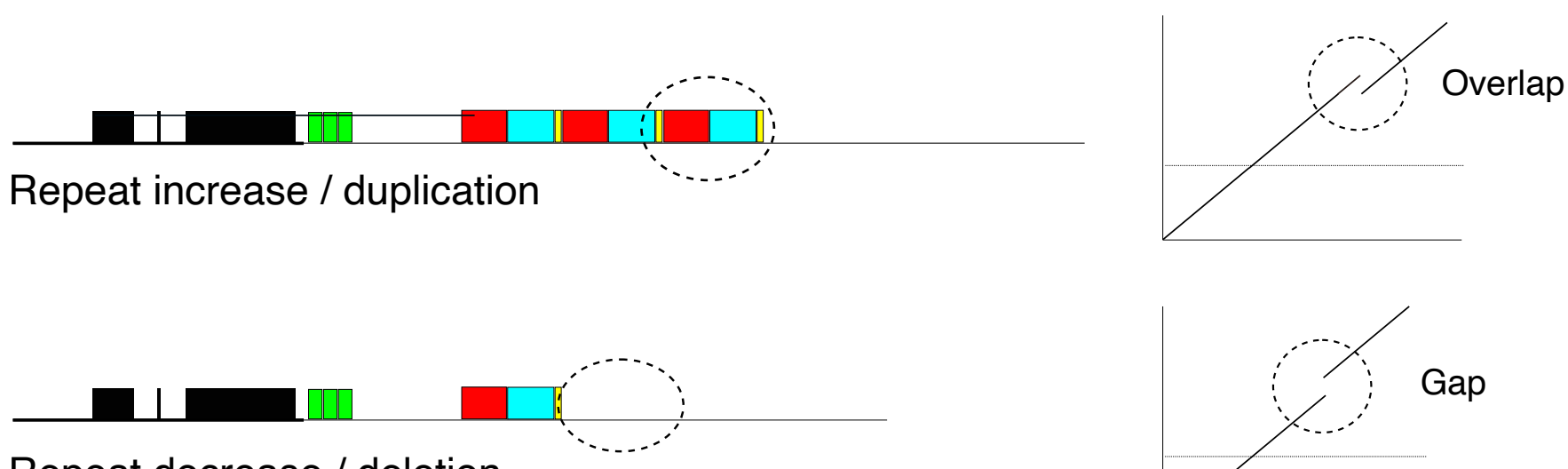

Repeat decrease / deletion
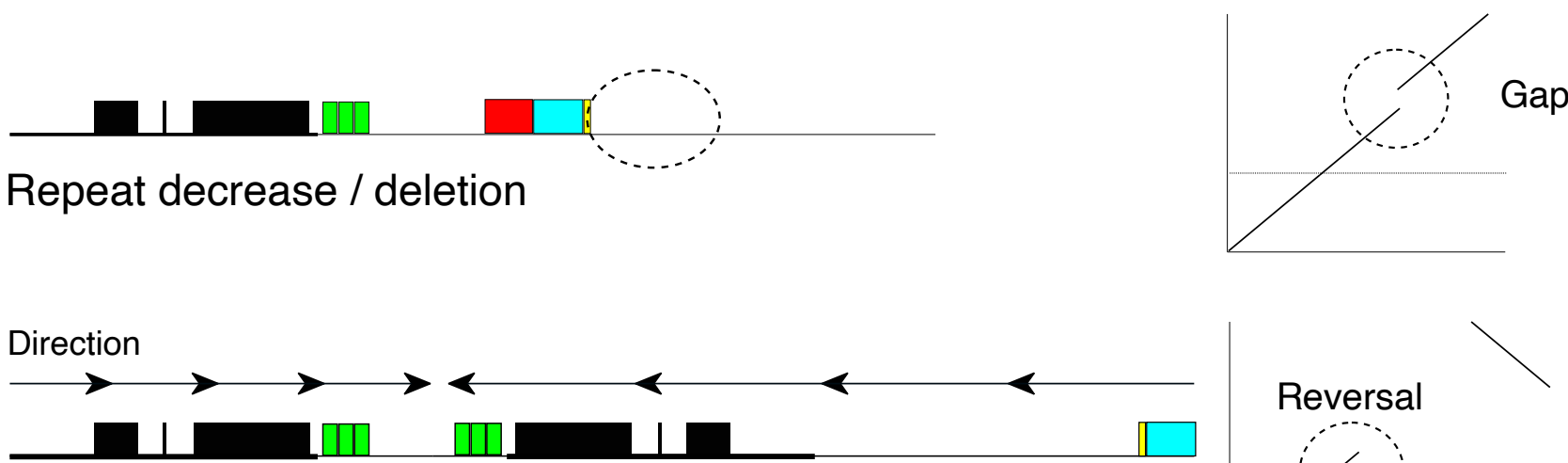

Inversion with short deletion

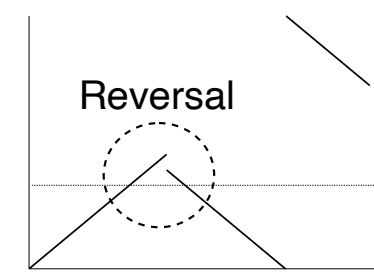

Expected visualization

Fig. 1 
Figure 1. rDNA structure and strategy for visualizing rDNA.

(A) Structure of rDNA. rDNA is largely divided into the coding 45S rRNA gene (45S rDNA) and the noncoding intergenic spacer (IGS). The IGS has repetitive sequences, such as microsatellite and transposable elements. Here, typical R and Long/Butterfly repeats are shown. (B) rDNA visualization strategy for Nanopore reads containing rDNA. First, the read is split into 300-nt sections, and each split read is mapped to the rDNA reference sequence. The structure is then reconstructed based on the position in the read and the mapped position in the reference. (C) Typical mutations and how they look in the visualization strategy.

genomic regions. For our in-house Cas9-enriched data, we analyzed the reads from DNA fragments in which both ends were digested with Cas9 RNP.

To determine the structure of rDNA, we developed a method that visualizes multiple copies of rDNA and the structural variation. In this method, the reads are split into $300 \mathrm{nt}$ and mapped to an rDNA reference sequence (GenBank accession KY962518.1) using BWA MEM aligner software suited for long read mapping (Li 2013; Kim et al. 2018). The shorter split length increases not only resolution but also the effect of sequencing errors of the Nanopore sequencer and reduces mapping frequency. By testing several lengths of split reads, we found that a 300-nt split is long enough to accomplish high mapping frequency (Supplemental Fig. S2A). Each 300-nt split read (short line) is plotted based on its location in the original read and its mapped position in the reference sequence (see Materials \& Methods; Fig. 1A, left panel). Therefore, when the reads are the same as the reference, a continuous straight line is generated (Fig. 1B, right panel). If there is a deletion, duplication or translocation, however, the line will be discontinuous (Fig. 1C). In selecting the reference sequence, we compared three different reference sequences of human rDNA

140 (GenBank accession KY962518, U13369, and AL592188) by counting the number of gaps between

141 successive split and mapped reads. If the distance between two neighboring mapped reads differed from the 142 expected distance (300 nt) by more than $100 \mathrm{nt}$, we considered that the pair was gapped and we counted the 143 number of gaps. We found that KY962518 had the least number of gaps for all samples and thus should be 144 the most typical rDNA sequence as the reference (Supplemental Fig. S2B) (Agrawal and Ganley 2018; Kim et al. 2018).

Fig. 2A shows actual representative data of a Cas9-enriched read. The read ( $40 \mathrm{~kb})$ had one copy of 147 rDNA with a gap in the Butterfly/Long repeat and a duplication in the R repeat region. Fig. 2B shows actual 148 data from HPGP WGS sequencing. The length of this read was $\sim 110 \mathrm{~kb}$, corresponding to two and a half tandem copies of rDNA, that is, IGS-45S-IGS-45S-IGS. From the visualization, we could identify that all of 

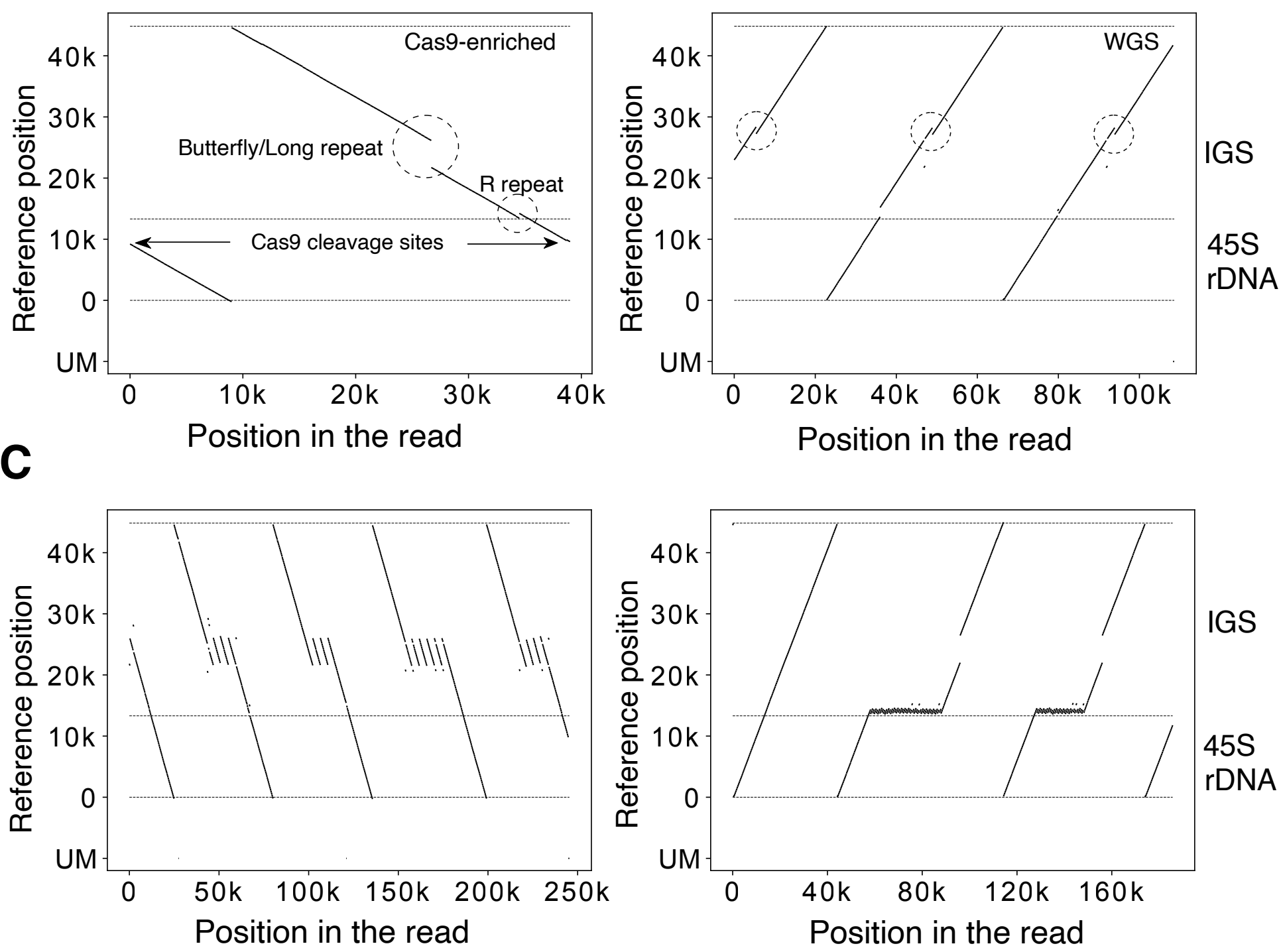

Fig.2 


\section{Figure 2. Visualization examples.}

(A)(B) Representative visualization pattern of a read obtained from Cas9-enrichment (A) and whole genome (B) sequencings. The vertical axis is the mapped position in the reference; the horizontal axis is the position in the Oxford Nanopore read. The horizontal dashed lines indicate the end of the reference and the border between $45 \mathrm{~S}$ rDNA and IGS. The unmapped split-reads are shown at the bottom (UM). The Sal box and the Butterfly/Long repeat region, which show variability among copies, are indicated (A). The same type of IGS variation is seen in all three rDNA copies (dashed circles) (B). (C) rDNA copies with an extremely long Butterfly/Long and R repeat. These sequences cannot be analyzed by short-read sequencers.

visualizing method is robust and should work in the structural analysis of rDNA. A strong point of the longread sequencer is that repeat structures of DNA can be analyzed; indeed, we were able to identify copies with extremely high repeat number variation in their IGS (Fig. 2C). In contrast, a weak point is that the fidelity of sequencing is not as high as that of short-read sequencers. But, as mentioned above, selecting an appropriate split length (300 nt) reduces this weakness and makes our structural analysis possible.

\section{$R$ and Butterfly/Long repeats are highly variable between copies}

By applying the split-and-map visualizing method to Nanopore data, we analyzed 39 samples (individuals) and identified variations in the Butterfly/Long and R repeat regions (Fig. 3A). By measurement of the Butterfly/Long repeat length of each read in each individual, we could classify the distribution into two types based on the proportion of copies that were more than 2,000 bases shorter than the reference (Fig. 3B; Supplemental Fig. 3A). These two types were also clearly differentiated by principal component analysis (PCA), confirming that our classification was not arbitrary (Supplemental Fig. S3B). In the shorter type, there were three discrete peaks and two of them were shorter than the reference. (e.g., HG03516). In the longer type (e.g., HG02080), the smaller peaks were not obvious in many cases, and more than $70 \%$ of copies were almost the same as the reference. Interestingly, all of the Japanese samples belonged to the shorter type (N=6: A0031, BSL2KA, PSCA0023, PSCA0047, PSCA0060, PSCA0517). Thus, there are differences among populations. In terms of the R repeat, the copy number varied from 0 to 4 (average 2-3 for most samples). As shown in Supplemental Fig. S4, the variation among samples was much larger for the $\mathrm{R}$ repeat than for the Butterfly/Long repeat and there were no clear differences among populations.

\section{Contiguous copies have similar variation patterns}


By comparing copies within reads that contain more than one copy of rDNA, the similarity between contiguous copies can be calculated. Specifically, we analyzed the differences in lengths of the R and Butterfly/Long repeat regions (Fig. 3C, upper panel). As a control, we also simulated the case where two random copies are compared (Fig. 3C, lower panel; Supplemental Fig. S5). In all samples, the distributions of length difference between contiguous copies were clearly shorter than the randomized simulated control in both regions. These observations indicate that contiguous copies are more similar than non-contiguous ones. Therefore, this suggests that gene conversion, at least, occurs locally and homogenizes the sequences of these repeats.

\section{The rDNA is quite regular in human cells}

192 Next, we analyzed larger-scale structural features of the rDNA. In a previous study, it was reported that 193 human rDNA contains many non-canonical irregular copies, such as palindrome structures (Caburet et al. 194 2005). Such irregular structures were suggested by a Fiber FISH (DNA combing) assay, in which long rDNA spread on a slide glass is hybridized with two fluorescent probes for different sites in the rDNA and fluorescence signals are detected by microscopy. As a result, uncanonical copies are identified by the irregularity of the aligned dotted signals. The study indicated that irregular rDNA copies account for $\sim 35 \%$ of total rDNA copies in a cell. Furthermore, this ratio was much increased in cells from Werner syndrome patients $(\sim 50 \%)$. structure of rDNA. First, we screened the reads in which more than $10 \%$ of the split reads were mapped in the opposite direction to the majority of the split reads and labeled them as inverted reads. Then, using the split-and-map visualizing data, we measured the distances between the splits, and compared them to the distance that was calculated from the reference sequence: if the repeat has an irregularly aligned or unusual structure, the distance between adjacent split reads will be larger and we can detect the difference. Unexpectedly, we found that reads with such large gaps are rare; that is, most of the rDNA copies were beautifully tandemly aligned on the chromosomes (Supplemental Table S1). In fact, the rate of non-canonical copies excluding palindromic reads in the healthy samples was less than $1 \%(0 \% \sim 0.7 \%)$. Furthermore, in $\sim 30 \%(12 / 39)$ of the individual samples, no non-canonical copies were detected. The most common 
A

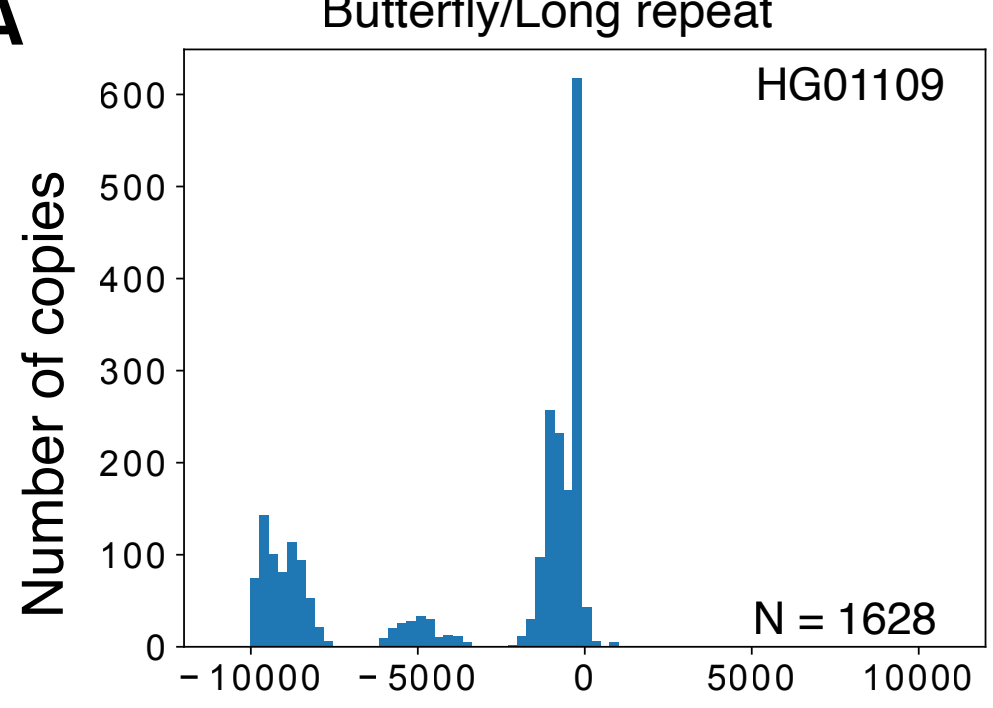

$\mathrm{R}$ repeat

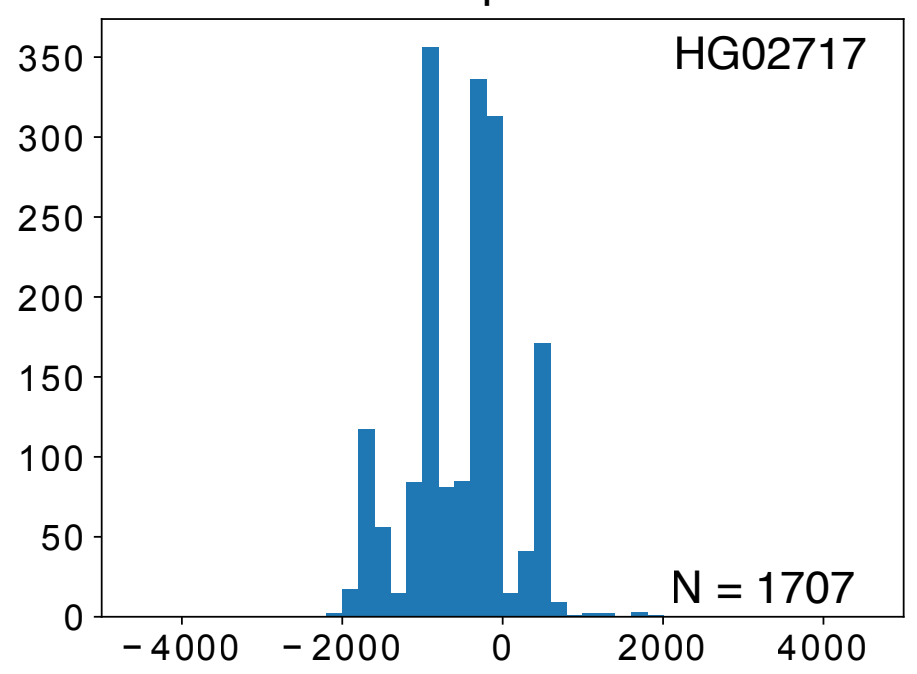

\section{Difference from the reference}

B

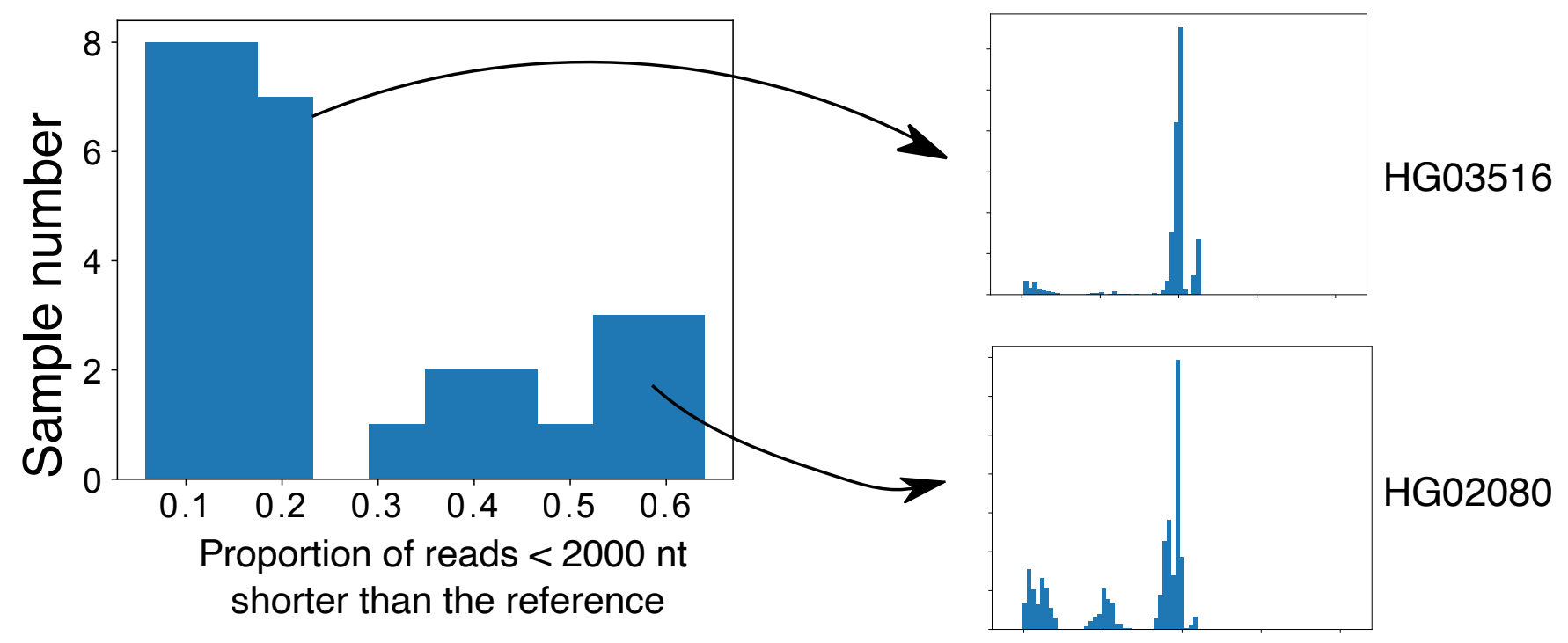

HG01109

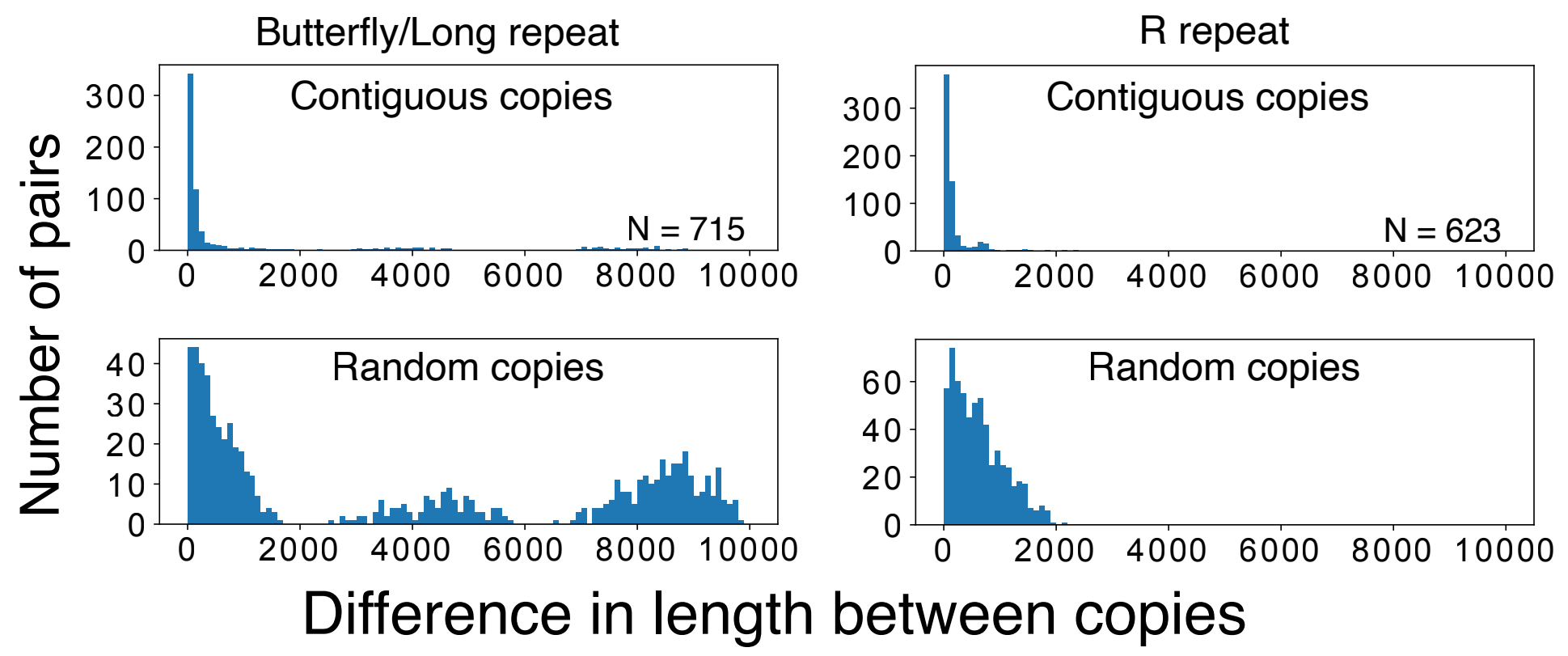


211 Figure 3. Variations in the IGS region.

212 (A) Length distributions of the variable Butterfly/Long and R repeats of the rDNA IGS in WGS samples. 213 Distributions are plotted based on the difference from the reference (0 indicates the reference length). In 214 these samples, repeat number variations can clearly be seen in the discrete peaks. (B) Plot showing the 215 proportion of reads with a Butterfly/Long repeat length shorter $(<2,000 \mathrm{nt})$ than the reference for each 216 sample. The samples can clearly be divided into two categories with each category having a similar pattern of size distribution among individuals (Supplemental Fig. 2). Typical distributions of long (HG03516) and short (HG02080) types are plotted on the right. (C) Differences in the length of contiguous reads calculated for the R and Butterfly/Long repeats (Upper panels). As a control, we calculated the differences in the length

structural mutation was duplication from the R repeat to the Long repeat region (Fig. 3A left; Supplemental

Table S1). We consider this type of mutation to be a common variation because it is limited to the IGS

region, where it is likely to have little impact on rRNA transcription. Another typical structural mutation was deletion (Fig. 4A right).

It should be noted that we found many palindromic reads, but they are thought to be artifacts for the

following reason. The Oxford Nanopore sequencer reads single-stranded DNA by separating double-stranded DNA at the pore (Fig. 4B). If the separation does not occur properly at the end of the first strand, sequencing of the second complementary strand may follow immediately (de Lannoy et al. 2018). Therefore, the resulting sequence read will look like a palindrome. Such "fake" palindromic reads should have their inversion site at the center of the read in cases where they were sequenced completely. A sequencing reaction may stop at any point in a read for various reasons. If it stops after the inversion, the inversion site should be in the latter half of the sequenced read. Taken together, if a palindromic read is an artifact, the inversion site will be at the center or in the latter half of the read. We therefore investigated the relative position of the inversion site in each palindromic read for HPGP samples. As shown in Fig. 4D, most of the inversion points appeared after the center, which strongly indicates that most of the palindromic reads are the result of the aforementioned artifacts. In addition, many such pseudo-palindromic reads showed a sudden drop in sequencing quality score around the inversion site. Some of the reads with an inversion site in the former half also showed a sudden drop in sequencing quality score, possibly meaning that they also are not real palindromes. Nevertheless, assuming that the reads with their inversion site in the first half are true palindromes, the estimated frequency of palindromic inversion is $\sim 1$ in 2000 copies. In summary, the human 
A

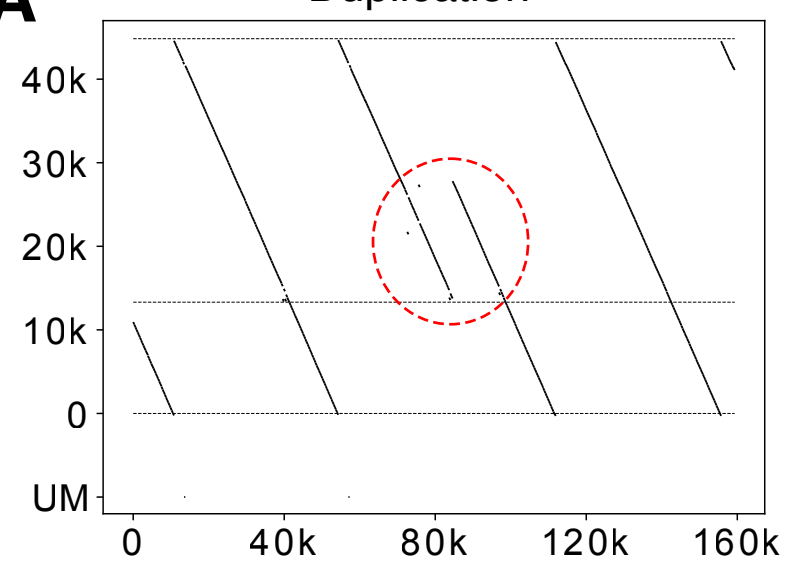

Deletion

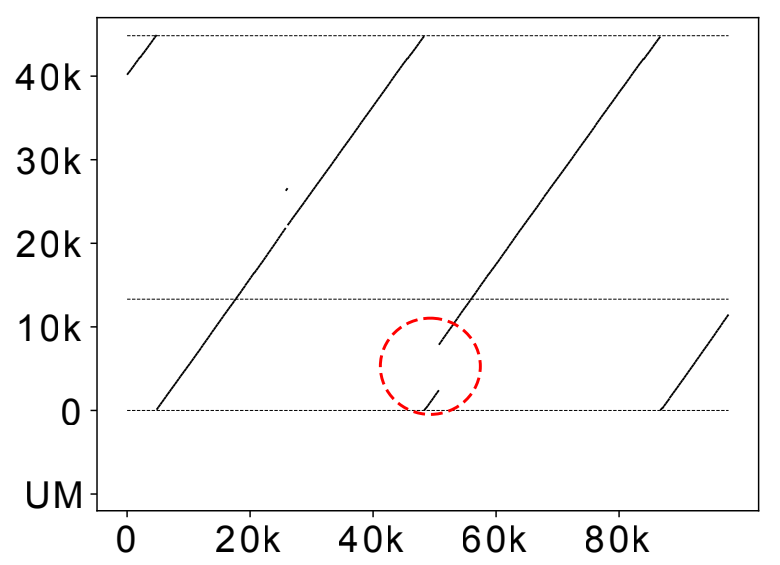

B

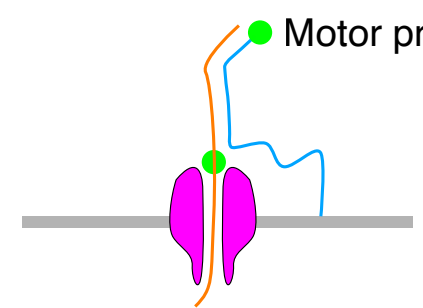

First strand sequencing

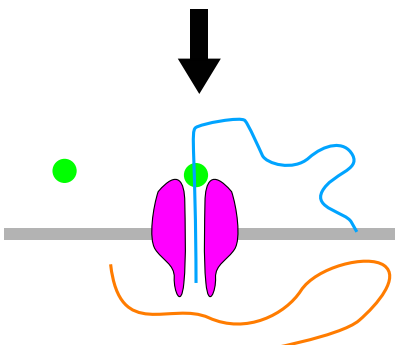

Immediate start of the second strand

C

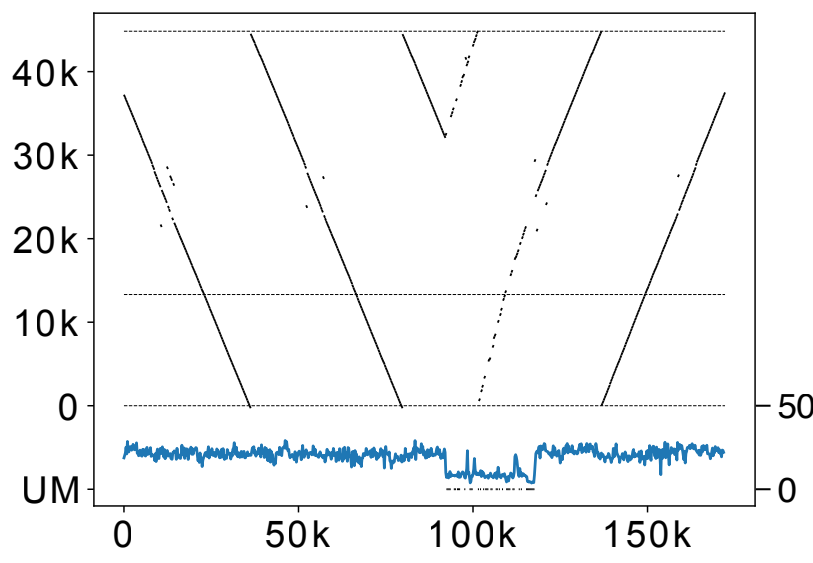

Both strands completely sequenced

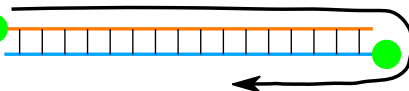

Sequencing stopping during the second strand

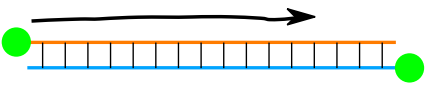

Sequencing stopping during the first strand

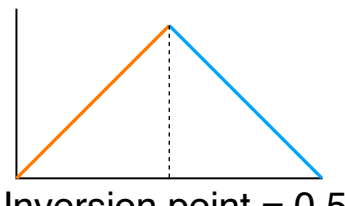

Inversion point $=0.5$

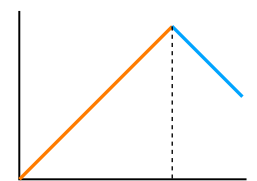

Inversion point $>0.5$

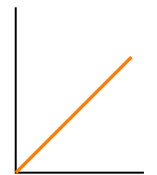

Not recognized as inverted

D

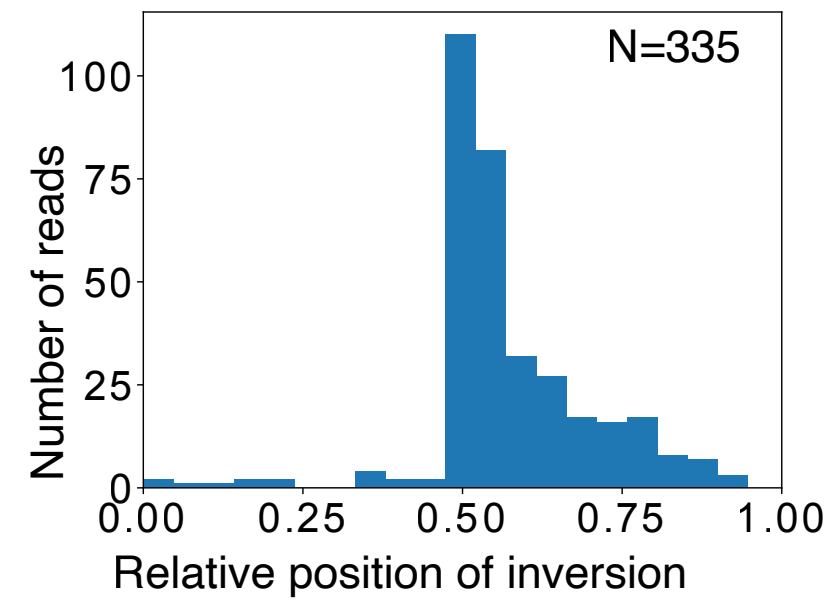


Figure 4. Large-scale structural variation in the rDNA array.

(A) Representative reads with large-scale variation in the rDNA array. (Left panel) The R to Butterfly/Long repeat region is doubled. (Right panel) A large portion of rDNA in the 45S rDNA is deleted. (B) The mechanism of Nanopore template switching that causes artifactual "fake" palindromic reads. (Left panel) After the completion of first strand sequencing, second (complementary) strand sequencing sometimes occurs. (Right panel) When sequencing terminates randomly after strand switching, the resulting distribution

\section{S rDNA is "all-or-none-methylated"}

Oxford Nanopore sequencing can also detect CpG methylation without any prior treatments. We therefore

investigated the rate of $\mathrm{CpG}$ methylation in the human rDNA. The methylation status of each read was calculated by binning (bin size $200 \mathrm{nt}$ ). We used two methods for calculating the methylation frequency of 45S rDNA in each bin: first, we calculated the expected value of the proportion of methylated bases by simply taking the mean of the posterior probability of each $\mathrm{CpG}$ being methylated that was output by Oxford Nanopore Guppy basecaller (Supplemental Fig. S6 "average"); and second, we estimated the proportion of

"threshold"). We obtained similar results by both methods; that is, the distinction between the methylated (>0.1) and less methylated ( 0.0) copies was clear in most samples except A0031 and HG03098. to be transcribed (Kass et al. 1997).

\section{Methylation in contiguous $45 S$ rDNA and the IGS is correlated}


In contrast to coding 45S rDNA copies, the non-coding IGS seems always to be methylated based on our visualization (Fig. 5A). Therefore, we investigated methylation status in the IGSs quantitatively. Because the Butterfly/Long repeat contains microsatellites with few $\mathrm{CpG}$ pairs and its length is variable, we excluded this region from the analysis. As a result, we found that nearly all of the IGSs are heavily methylated (average 51\%) (Fig. 5C; Supplemental Fig. S7). Furthermore, we evaluated the methylation rate of 45S rDNA and its contiguous IGS and found that the rate of methylated bases in the IGS was correlated with the methylation level of contiguous $45 \mathrm{~S}$ rDNAs in many individuals when the calculation was limited to strongly methylated 45S rDNA (>0.3), although the strength of correlation differed among samples (Fig. 5D; contiguous IGS together form heterochromatin.

\section{Contiguous 45S rDNAs have a similar CpG methylation pattern}

289 Using information in large reads containing several rDNA copies, we analyzed the relationship of methylation status among contiguous $45 \mathrm{~S}$ rDNAs (Fig. 5E; Supplemental Fig. S8). Interestingly, we found that contiguous $45 \mathrm{~S}$ rDNA copies have a similar $\mathrm{CpG}$ methylation pattern as compared with noncontiguous random copies. In other words, unmethylated $45 \mathrm{~S}$ rDNAs form clusters. This suggests that heterochromatin structure is present in the large rDNA region and the transcription of rDNA is inhibited in this region. suggested that the transcriptional state of rDNA is determined at the chromosome level (Roussel et al. 1996).

We found that a contiguous pair with different methylation status occurs at the frequency about 1 in 20 pairs in many individuals (Supplemental Table S3). Therefore, chromosomes that have more than 20 copies of rDNA should have, on average, more than one change in methylation status. This speculation is supported by a recent study (van Sluis et al. 2020).

\section{The number of $R$ repeats is not related to rDNA methylation}

302 Next, we tested the correlation between 45S rDNA methylation rate and the copy number of the R repeat that 303 is associated with TTF1 (Grummt et al. 1986). Notably, Spearman correlation between the 45S rDNA methylation rate and $\mathrm{R}$ repeat copy number differed among individuals (21/32 individuals showed $>0.05$ 
A

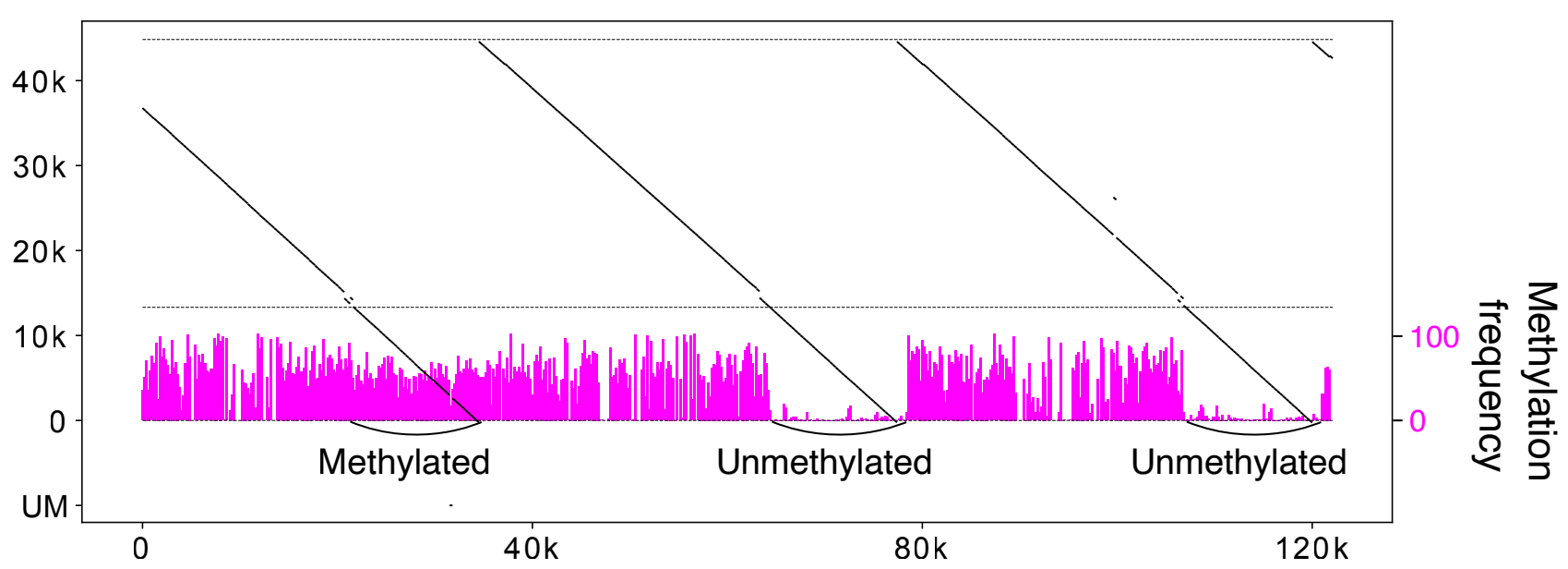

B

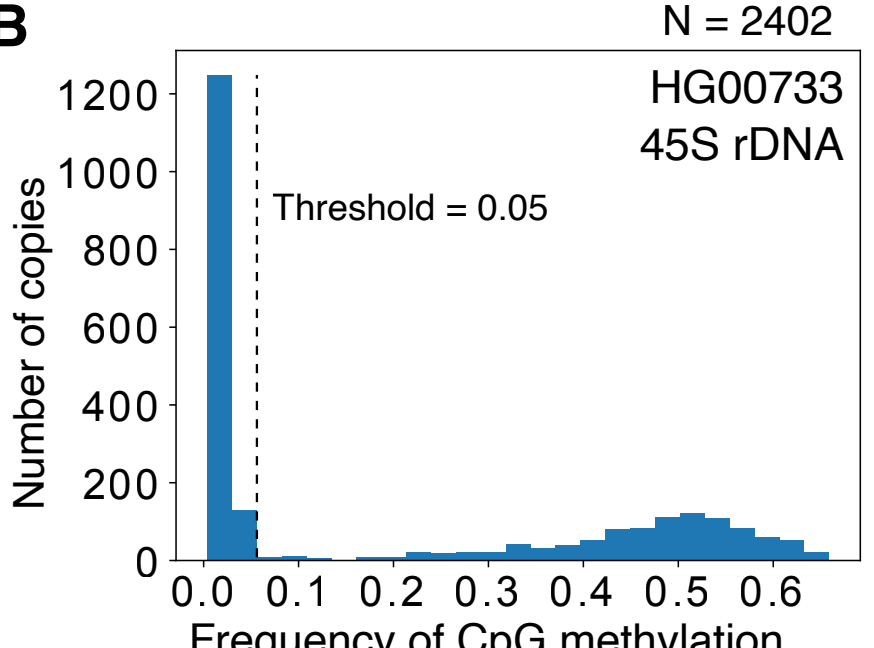

D

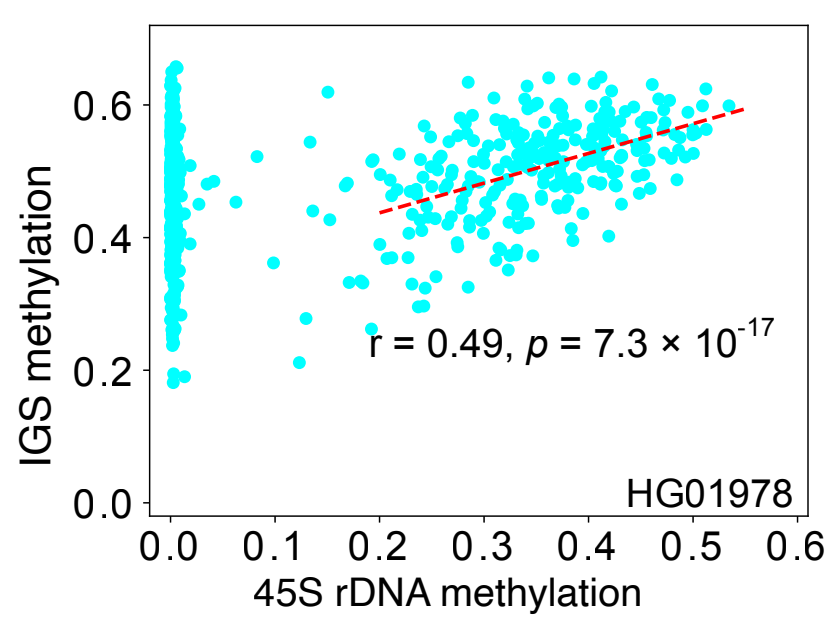

$\mathbf{F}$

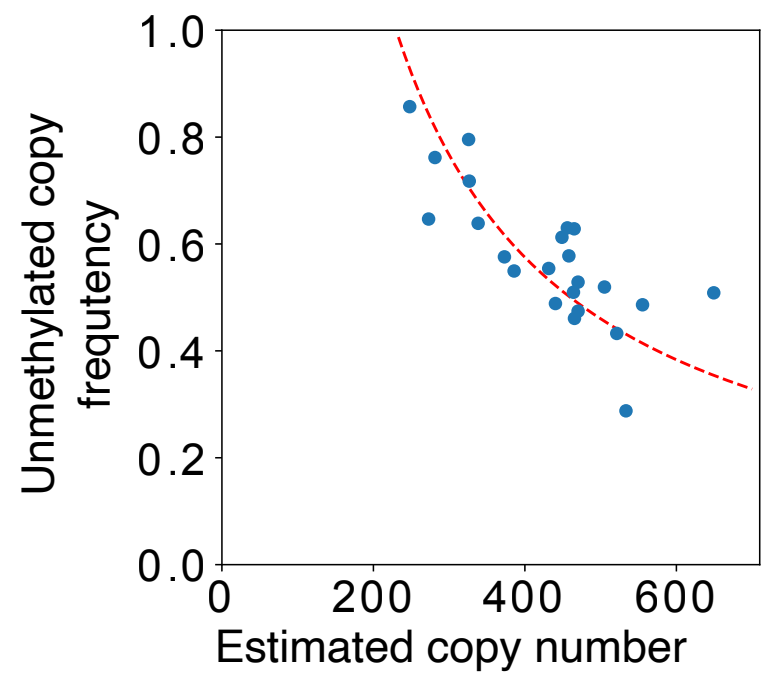

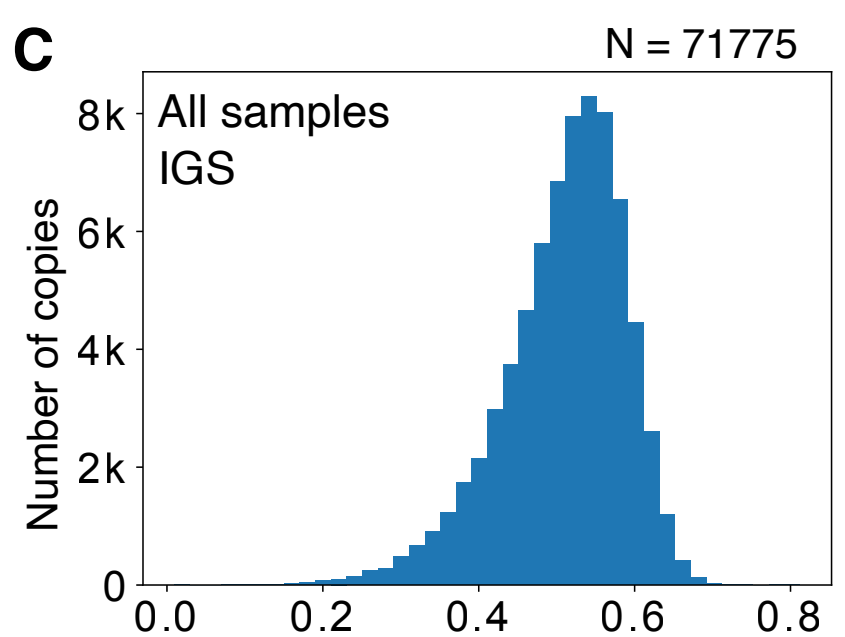

Frequency of $\mathrm{CpG}$ methylation
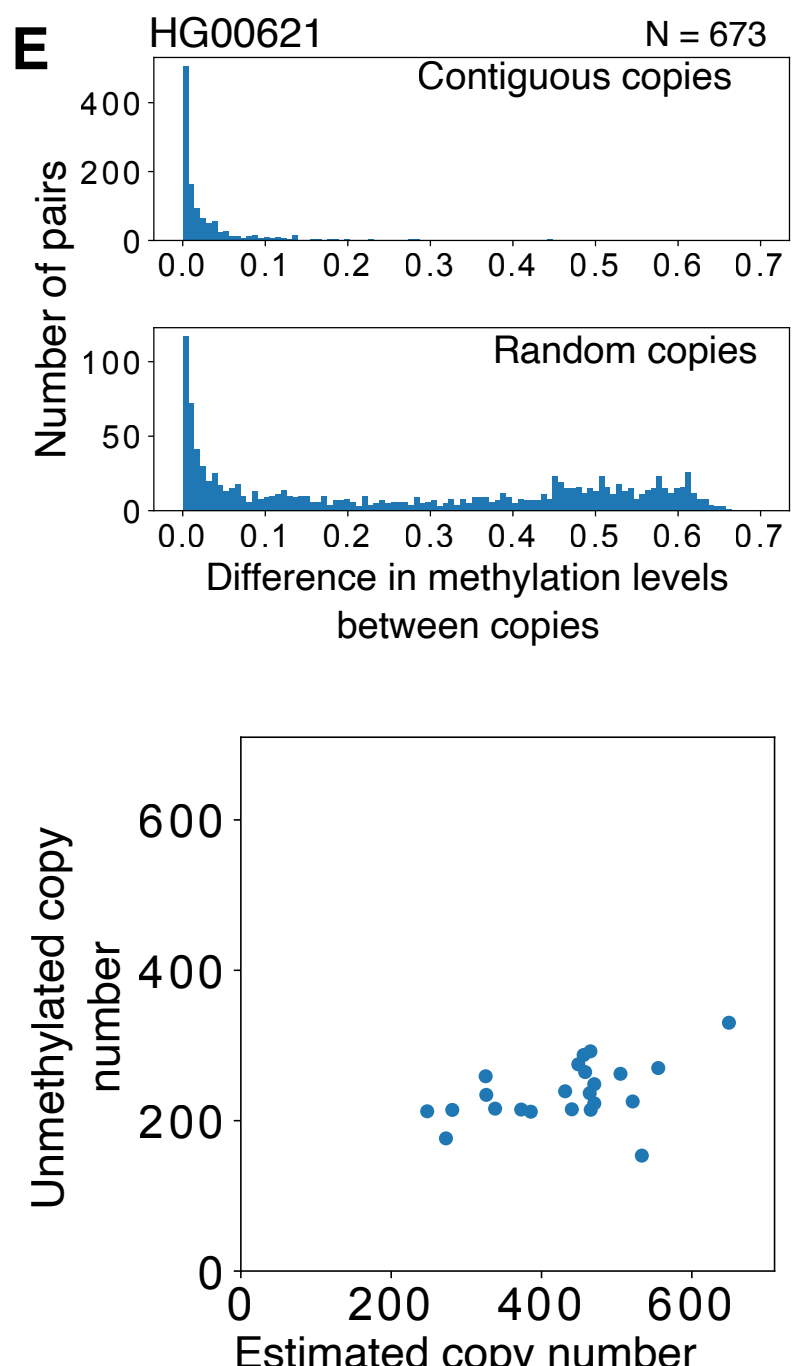

Fig.5 
Figure 5. Methylation analysis of rDNA.

(A) Representative visualization of CpG methylation. Reads are split into 200-nt bins and the expected frequency of $\mathrm{CpG}$ methylation is calculated for each bin using posterior probability output by Guppy basecaller. The methylation frequency of each bin is shown as a vertical blue bar. In the read shown, both the methylated and less methylated 45S rDNA are included. Note that the three IGS methylation patterns are similar despite the difference in the $45 \mathrm{~S}$ rDNAs. (B) Average proportion of methylated $\mathrm{CpG}$ for each $45 \mathrm{~S}$ rDNA (calculated by taking the mean of posterior probabilities), and distribution in the HG00733 sample. Dashed line indicates the border between the less methylated copies and methylated copies. (C) Proportion of CpG methylation in the IGS in all samples. Most of the IGS copies are heavily methylated. (D) Methylation level of 45S rDNA and its flanking IGS. In many samples, there is a clear correlation for copies with highly methylated $45 \mathrm{~S}$ rDNA (dashed line). (E) Differences in the methylation levels of contiguous $45 \mathrm{~S}$ rDNAs and randomized controls. The methylation pattern is similar between adjacent copies of contiguous 45S rDNA, as in the case of repeat number variation in the IGS (Fig. 3C). (F) Relationship between the estimated rDNA copy number and the proportion of unmethylated copies (left) or the estimated number of unmethylated copies (right). Dashed line in the left panel is a theoretical line based on the assumption that the number of active rDNA copy is constant at 230 .

individuals and both positive and negative correlations were observed (Supplemental Fig. S9, Supplemental

Table S4). We speculate that the correlations observed in some samples were the effect of the correlation of contiguous copies; thus, they do not reflect a true correlation. Collectively, these observations suggest that the $\mathrm{R}$ repeat plays little role in the transcription of rRNA.

\section{There is no strong correlation between age, instability and methylation}

Next, we sequenced two samples from young individuals (20s) and two from older individuals (70s) to investigate age-related changes in rDNA structure (Supplemental Table S1, row\# 4-7). Between the Cas9enriched young and old samples, no large-scale structural differences were observed. This is consistent with the previous finding that aging does not increase such differences (Caburet et al. 2005).

It has been proposed that the rate of methylation of $45 \mathrm{~S}$ rDNAs is increased with age (Watada et al. 2020; Wang and Lemos 2019). We therefore tested this relationship using the two young samples and two nor the average methylation level of methylated copies was increased in the older samples (Supplemental Table S1). It should be noted that we did not analyze methylation status in the same individual over time and that the number of samples was not sufficient to draw conclusions, which may explain why our results were different from previous studies. 

rDNA copy number per cell, we used the ratio of rDNA reads to the total reads in each sample. By this calculation, the rDNA copy number ranged from 250 to 700 copies per cell (Supplemental Table S5; Fig. as quantitative PCR and short-read high-throughput sequencing (Malinovskaya et al. 2018; Parks et al. 2018).

To analyze the relationship between rDNA copy number and methylation rate, we used only the HPGP data that were generated by the Human Pangenomics Reference Consortium (HPRC), which were all thought to be obtained around the same period and basecalled with Guppy 4.0.11 (see Methods). This was done to avoid artifacts caused by different library preparation and sequencing conditions. From the analysis of 23 HPGP samples, we found that the number of rDNA copies and the ratio of unmethylated copies per cell were negatively correlated (Pearson correlation, $\mathrm{r}=0.749, p=1.15 * 10^{-5}$ ). In other words, the number of

\section{rDNA instability is increased in progeroid syndrome}

We also analyzed two cell lines derived from patients with progeroid syndrome: namely, Bloom syndrome patient B cells derived by EBV transformation, and Werner syndrome patient primary fibroblast cells. These syndromes are caused by mutations in the DNA repair machinery, which increases genome instability (Killen et al. 2009). A previous study using the Fiber FISH method suggested that the structure of rDNA is highly aberrant ( $\sim 50 \%$ of total rDNA copies) in Werner syndrome patients (Caburet et al. 2005). Based on the Cas9enriched Oxford Nanopore sequencing method, the rate of non-canonical (e.g., real palindrome) copies was $1.2 \%$ and $2.4 \%$ in Werner and Bloom patient cells, respectively. These values are much higher than those in the normal samples $(\sim 0.2 \%)$, but much lower than the previously reported value $(\sim 50 \%)$. In both progeroid syndrome samples, we found characteristic reads, including a duplication within the 45S rDNA that may mutations were concentrated around the 7,000-14,000-nt region in the reference. Because they were relatively rare in the other samples (Supplemental Table S1, column 7), we speculate that they are genomic instability "hotspots", where mutation is frequent in DNA repair compromised cells. 


\section{Human pluripotent stem cells have a different methylation status}

Next, we analyzed rDNA methylation status in human induced pluripotent stem cells (hiPSCs, 201B7) by Cas9-enriched sequencing. In ESCs and iPSCs, rDNA is thought to be globally unmethylated because of the high transcription activity (Gupta and Santoro 2020). Unexpectedly, however, around half of the $45 \mathrm{~S}$ rDNAs were methylated and the IGS was heavily methylated in the iPSCs, similar to differentiated cells (Fig. 6A; Supplemental Fig. S11, see Discussion). We also tested iPSCs derived from Werner syndrome patient fibroblast cells (A0031) (Shimamoto et al. 2014). Similarly in these iPSCs, a substantial proportion of 45S rDNAs were methylated and the frequency was higher than in the original A0031 fibroblast cells (52\% vs $43 \%, p \approx 1.2 * 10^{-6}$, Fisher's exact test, Fig. $\left.6 \mathrm{~B}\right)$, in contrast to the results of previous studies

381 (Woolnough et al. 2016; Wang and Lemos 2019).

In terms of rDNA stability, the frequency of aberrant structures was found to be significantly decreased in A0031-derived iPSCs ( $p \approx 0.007$, Fisher's exact test, Supplemental Table S1). This is consistent with the finding that iPS induction suppresses chromosomal instability (Shimamoto et al. 2014), and we speculate that cells with stable genetic information are selected during the iPS induction process. If this is the case, the increased methylation of the transcribed region might be due to the selection process.

rDNA structure and methylation in mouse

To test the generality of the Nanopore sequencer, we also analyzed two mouse-derived samples (bone marrow cells extracted from femur of an 8-week-old male C57BL/6J mouse and feeder-free ht7 embryonic stem cells (ESCs) derived from 129/Ola strain) (Niwa et al. 2000). Although mouse ribosomal DNA has been said to be around $45 \mathrm{~kb}$ in length (Grozdanov et al. 2003), we found that the average estimated size was much shorter than previous reported at $39.5 \mathrm{~kb}$ and $38 \mathrm{~kb}$ for $\mathrm{C} 57 \mathrm{BL} / 6 \mathrm{~J}$ and ht7, respectively. Mouse rDNA also has variation in repeat number in the IGS, and this seems to determine the size distribution of rDNA (Fig. 6C; Supplemental Fig. S12).

In terms of methylation, $45 \mathrm{~S}$ rDNAs of mESCs were almost completely free from methylation, 
A bioRxiv preprint doi: https://doi.org/10.1101/2021.06.02.446762; this sion posted June 3, 2021. The copyright holder for this preprint (which was not certified by peer review) is the author/funder. Mrights reserved. No reuse alloweg withputparmission.

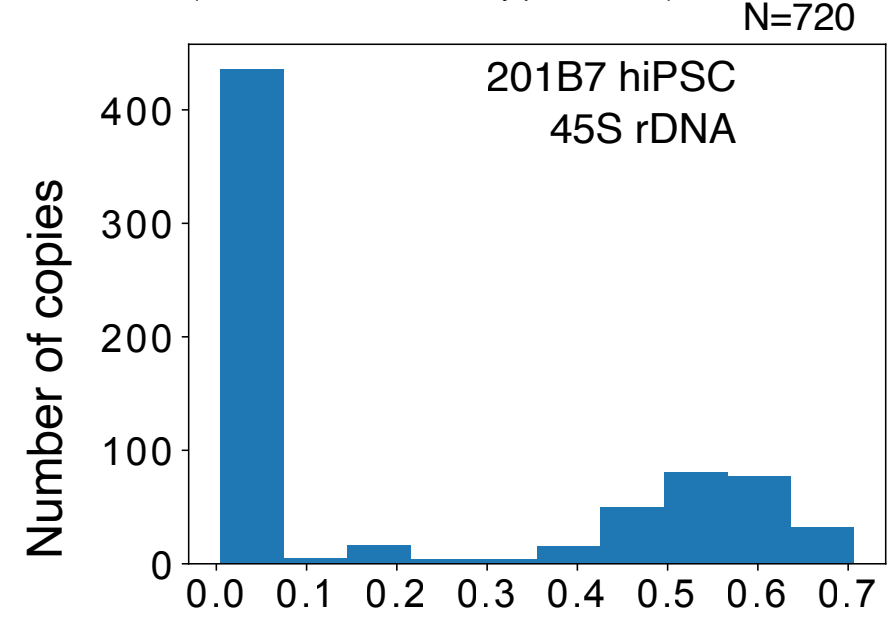

Frequency of $\mathrm{CpG}$ methylation

C

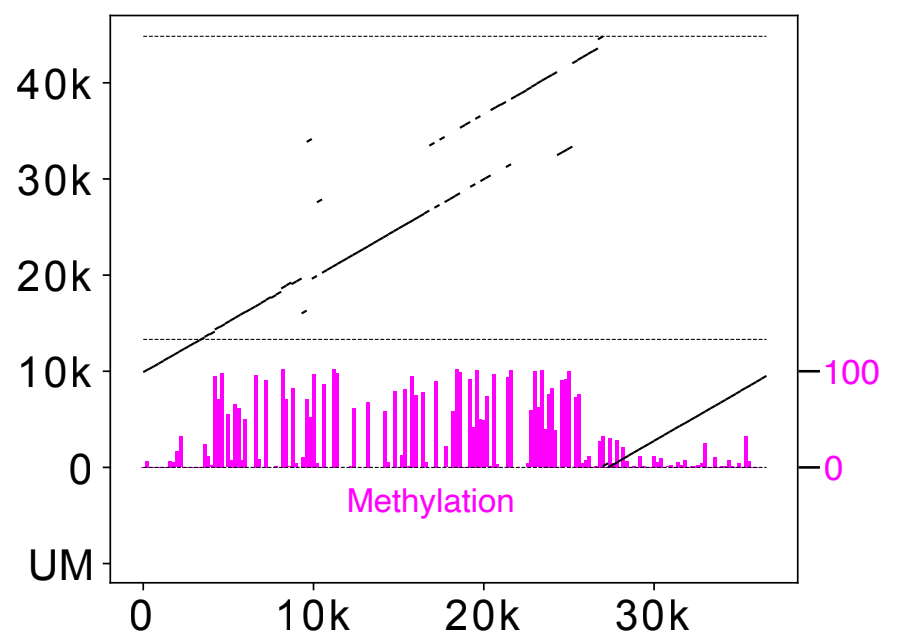

D

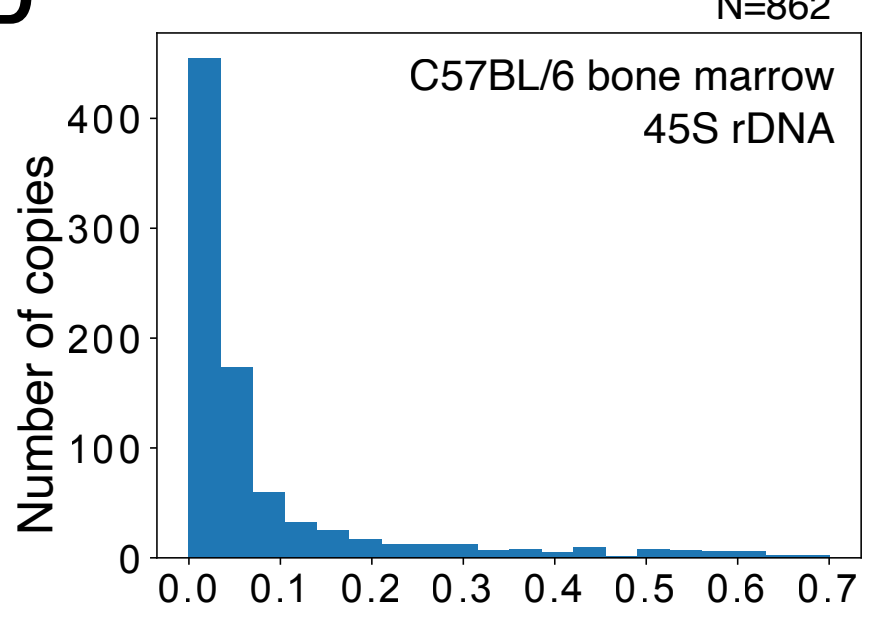

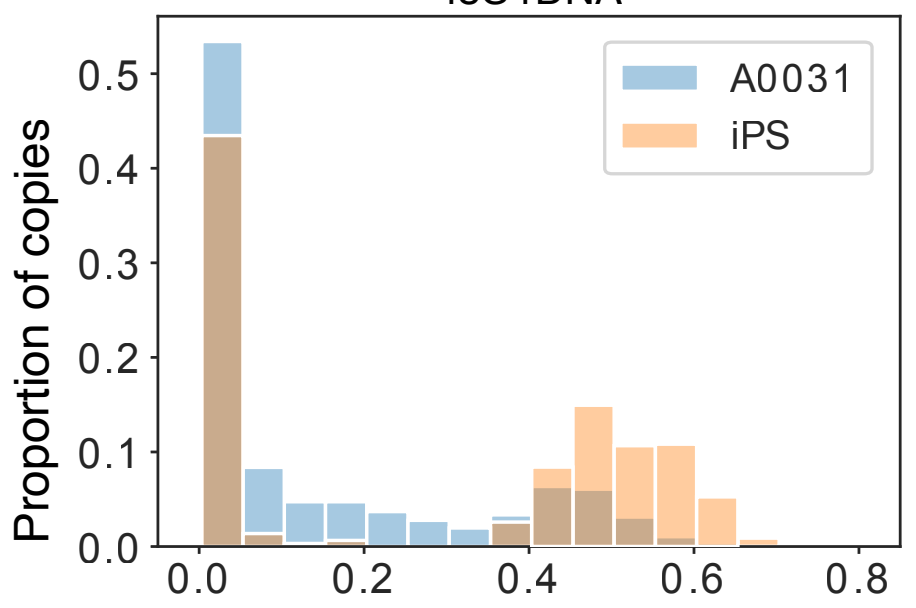

Frequency of $\mathrm{CpG}$ methylation
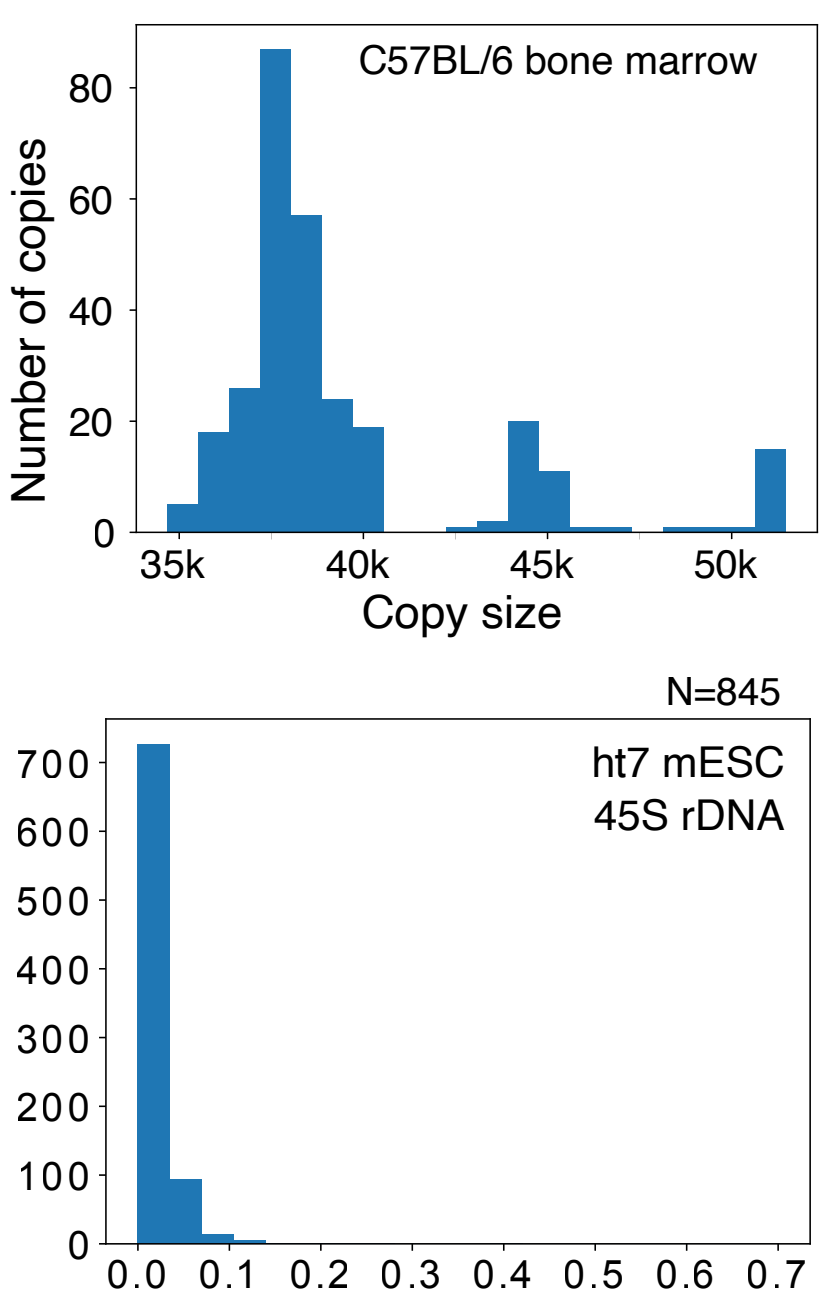

Frequency of $\mathrm{CpG}$ methylation

E

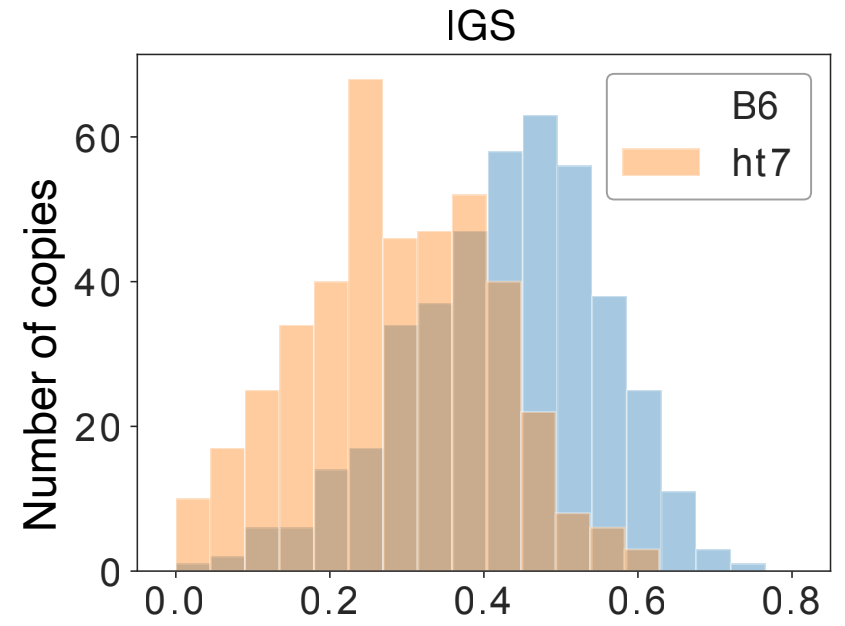

$\mathbf{F}$

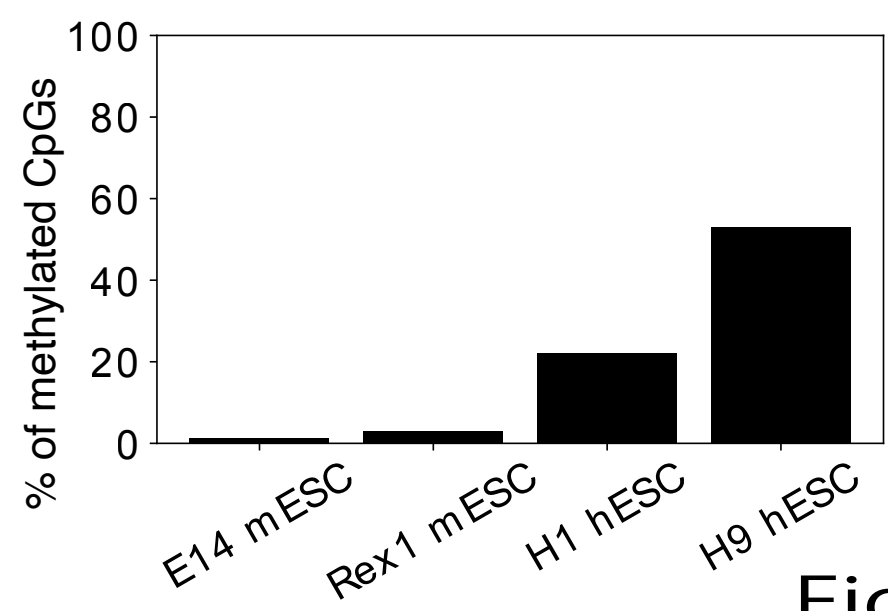

Frequency of $\mathrm{CpG}$ methylation 
Figure 6. rDNA methylation of pluripotent stem cells.

(A) The 45S rDNA methylation status of rDNA in hiPSCs does not differ from that in other differentiated samples: $\sim 40 \%$ of the transcribed region is methylated. (B) Comparison of the $45 \mathrm{~S}$ rDNA methylation status in Werner syndrome patient fibroblasts (A0031) and iPSCs derived from them. The $y$ axis is the proportion of reads in each bin. The frequency of methylated copies is increased in iPSCs. (C) Representative rDNA from a Cas9-enriched Nanopore read of a mouse sample (left panel). Magenta bars represent CpG methylation. The estimated size distribution of mouse rDNA copies in C57BL/6 bone marrow cells is shown on the right. Note that the rDNA copy size of mouse is much smaller than previously reported. (D) $45 \mathrm{~S}$ rDNA methylation levels in B6 bone marrow cells and ht7 mESCs. Methylation levels among copies are more continuous in mice and almost no methylation is seen in mESCs. (E) Comparison of IGS methylation levels in B6 bone marrow cells and ht7 mESCs. ht7 clearly shows lower a methylation level, even in the IGS region. (F) Proportion of CpGs methylated in the 45S rDNA of mESCs and hESCs determined by using

were clearly methylated but, unlike in human, the distribution of $\mathrm{CpG}$ methylation among copies was continuous rather than bimodal, making it difficult to clearly define methylated copies (Fig. 6D left). The IGS was also methylated in mouse, but the frequency of methylation was much lower in mESCs than in hiPSCs (Fig. 6E). To examine whether the difference in methylation status between hiPSCs and mESCs was the result of the derivation method (i.e., ES vs iPS) or related to species differences (i.e., human vs mouse), we re-analyzed publicly available short-read-sequencer data, which showed that $45 \mathrm{~S}$ rDNAs of mESCs are rarely methylated but a substantial proportion of CpGs in hESCs are methylated (Fig. 6F). Thus, the

difference is likely to be ascribed to species variations. In fact, mESCs are known to be in a more

undifferentiated state as compared with hESCs and show global hypomethylation (Nichols and Smith 2009;

\section{DISCUSSION}

In this study, we analyzed the long rDNA array using data from the Oxford Nanopore sequencer. Our findings provide a new picture of the rDNA structure based on 39 samples with many reads that were directly extracted from human cells ( $\sim 78,000$ copies, more than 3 billion bases).

By using a new method that visualizes multiple copies of rDNA, we first characterized the rate of

431 large-scale rDNA instability (inversion, deletion and other non-canonical structures) and found that such 432 mutations are relatively rare, contrary to a previous report based on the Fiber FISH method (Caburet et al.

433 2005). We also showed that Oxford Nanopore sequencing occasionally produces artifactual palindromic 434 reads that are considered to be difficult to distinguish from true palindromic reads (de Lannoy et al. 2018). 
435 Fortunately, we found such artificial palindromic reads have characteristic features, such as a poor quality 436 score around the inversion and the position of the inversion site, and succeeded in recognizing them. As a 437 result, we conclude that real palindromic structures are relatively rare. Lastly, we found that, although rDNA 438 instability was not increased by age in our samples, it was increased in cells from patients with progeroid 439 syndrome. In our study, 440 samples were analyzed and only $0.2 \%$ (on average) of structures were non440 canonical. This value is much lower $(\sim 1 / 30)$ than the value previously reported using Fiber FISH (Caburet et 441 al. 2005). Thus, we conclude that the human rDNA is a relatively regular array.

We found that the R and Butterfly/Long repeat regions are variable in different copies, although they are similar in contiguous copies. Because the $\mathrm{R}$ and Butterfly/Long repeat regions have small repetitive sequences within the repeats, they may form a secondary DNA structure and inhibit the replication folk to induce instability. The R repeat contains many copies of the Sal box associated with TTF1 (Fig. 1A), which is known to arrest the replication fork both in vivo and in vitro (Santoro and Grummt 2005; Akamatsu and Kobayashi 2015). This sequence may work as a recombination hotspot, as observed in budding yeast (Supplemental Fig. S1; see below).

In contrast to variation, our study indicated that there is structural similarity between IGSs in contiguous copies. This suggests that gene conversion takes place frequently in the human rDNA, as in the budding yeast rDNA (Ganley and Kobayashi 2011). Previous studies also have suggested the possibility of IGS homogenization within the same chromosome (Gonzalez and Sylvester 2001). Our study strongly supports this view.

We also found that IGS are classified into two types among individuals (Fig. 3B; Supplemental Fig. S3). The mechanism behind this bimodal distribution of Butterfly/Long repeat copy number is a fascinating issue to be addressed in future studies. Another mystery is the presence of a rare type of IGS, which was observed in many samples. If the efficiency of gene conversion is high, such copies should be excluded. One explanation for the rare-type IGS is that these variations are often generated by chance and resolved over time by homogenization.

In the budding yeast, it is known that gene conversion occurs frequently and all copies essentially 461 have the same sequence in a cell (Ganley and Kobayashi 2007). As a mechanism, Fob1/RFB-dependent rDNA recombination seems to be important (Ganley and Kobayashi 2011). As mentioned above, such an 
may also contribute to sequence homogenization in human cells (Akamatsu and Kobayashi 2015). Moreover, in progeroid syndrome patient cells, in which the activity of DNA repair is reduced, the number of noncanonical copies increased. Such rDNA instability has been also observed in the yeast WRN homologue mutant sgs 1 (Sinclair and Guarente 1997).

In terms of rDNA methylation, we found that there is an obvious difference between methylated and unmethylated 45S rDNAs (Fig. 5A,B). In unmethylated 45S rDNAs, the methylation rate was close to 0 such that the genes are likely to be transcribed (reviewed in Kass et al., 1997). The methylation status was also similar in contiguous copies. This may be because heterochromatin forms around these regions and affects rDNA silencing. In contrast to the "all-or-none" methylation pattern of 45S rDNAs, the non-coding IGS regions were always methylated, and the level was correlated with the methylation level of the contiguous 45S rDNAs (Fig. 5D). These observations suggest that the IGS is always in a similar heterochromatin structure and that $45 \mathrm{~S}$ rDNA activation affects the region. We also have to note that, because all of the IGS regions in the rDNA are heavily methylated, the transcriptional rate of non-coding sequences in these regions may not be very high. Therefore, if this is the case, at least some non-coding transcripts are like to come from rDNA fragments that are scattered all over the human genome (Cherlin et al. 2020).

We found that unmethylated copies are negatively correlated with total rDNA copy number in a cell, suggesting that the number of unmethylated copies is roughly constant in different individuals, at least in the same tissue. This finding also supports our definition of unmethylated copies and our view that they are the actively transcribed ones. One possible mechanism behind the regulation of active copy number is that the transcription factor dosage is limited. Alternatively, the volume of the nucleolus fibrillar center may be kept at a constant level, which restricts the number of rDNA copies that can be held inside. Further analysis will be required to reveal the underlying mechanism.

In terms of mouse rDNA, we found that some features were similar to the human rDNA, such as repeat number variation in the IGS. One clear finding was that the unit length was shorter $(\sim 39 \mathrm{~kb})$ than reported previously ( $\sim 45 \mathrm{~kb}$ ) (Grozdanov et al. 2003). The reason might be the difficulty in assembling sequences filled with repeats using relatively short reads.

Lastly, regarding the methylation in ES and iPS cells, the results differed between human and mouse.

We expected that both cell types would be less methylated than their differentiated counterparts because the nucleolus of ES and iPS cells is known to be larger and rRNA transcription activity is high (Woolnough et 
al. 2016; Gupta and Santoro 2020; Wang and Lemos 2019). In the human iPSC and ESCs, however, the methylation status of the $45 \mathrm{~S}$ rDNA was similar to that in differentiated cells. In contrast, most of the $45 \mathrm{~S}$ rDNA copies in the mouse ESCs were unmethylated. This difference between mouse and human is thought to stem from the difference between their developmental stages. Mouse ESCs are always in a pre-Xchromosome-inactivated status and are globally hypomethylated; in human ESCs, by contrast, the Xchromosome is already inactivated in many cases and the other genomic regions are also highly methylated (Tomoda et al. 2012; Nishino and Umezawa 2016; Nichols and Smith 2009). In summary, our results have revealed several new aspects of the most highly transcribed house-

METHODS

\section{DNA extraction}

DNA was extracted by modified Sambrook and Russell DNA extraction. In brief, $1 \times 10^{6}$ cells were pelleted and $500 \mu \mathrm{L}$ of TLB was added (10 mM Tris-Cl pH 8.0, $25 \mathrm{mM}$ EDTA pH 8.0, 0.5\% (w/v) SDS, $20 \mu \mathrm{g} / \mathrm{ml}$ RNase A). After mixing by inversion and incubating for 1 hour at $37^{\circ} \mathrm{C}, 1 \mu \mathrm{L}$ of $20 \mathrm{mg} / \mathrm{ml}$ Proteinase $\mathrm{K}$ (Roche) solution was added and the mixture was further incubated for 3 hours at $55^{\circ} \mathrm{C}$. Next, $500 \mu \mathrm{L}$ of TEsaturated phenol was added and the solution was rotated until the water phase was clear. Phase lock gel (Dow 510 Corning(R) High Vacuum Grease) was added, followed by centrifugation for $5 \mathrm{~min}$, and then the upper phase 511 was decanted into a 1.5-mL tube. Phenol/chloroform/isoamyl alcohol (25:24:1) was added and the above 512 procedure was repeated. The resultant solution was placed in a 5-mL tube, and $200 \mu \mathrm{L}$ of $5 \mathrm{M}$ ammonium 513 acetate and $1.5 \mathrm{~mL}$ of $100 \%$ ethanol were added with gentle rotation at RT until the solution was 514 homogeneous. The DNA precipitate was collected by pipetting and placed in a $1.5 \mathrm{~mL}$ tube containing $70 \%$ ethanol. After centrifugation, $50 \mu \mathrm{L}$ of TE was added to the pellet, which was left overnight at $4{ }^{\circ} \mathrm{C}$. DNA concentration was measured with a Qubit assay kit (Invitrogen).

\section{Construction of the Cas9-enriched Oxford Nanopore library}

519 The published protocol (Gilpatrick et al. 2020) was modified specifically for rDNA, which consists of 520 hundreds of copies. First, Cas9 RNP was assembled as described previously. Then, 500 ng of DNA was 
pipette set at $8 \mu \mathrm{L}$ with a P2/P10 tip. One microliter of QuickCIP (New England Biolabs) was added and the solution was incubated at $37^{\circ} \mathrm{C}$ for $10 \mathrm{~min}$, followed by $80^{\circ} \mathrm{C}$ for $2 \mathrm{~min}$. Next, $0.5 \mu \mathrm{L}$ of RNP, $0.3 \mu \mathrm{L}$ of 10 $\mathrm{mM}$ dATP and $0.3 \mu \mathrm{L}$ of Taq DNA polymerase were added to the solution before incubation at $37^{\circ} \mathrm{C}$ for 15 $\min$ and $72{ }^{\circ} \mathrm{C}$ for $5 \mathrm{~min}$. The ligation mix ( $5 \mu \mathrm{L}$ of LNB, $3 \mu \mathrm{L}$ of Quick ligase (New England Biolabs), 1.2 $\mu \mathrm{L}$ of $\mathrm{MQ}, 0.8 \mu \mathrm{L}$ of AMX) was then added to the DNA solution in two stages, with tapping to mix between additions. After incubating for $10 \mathrm{~min}$ at room temperature, $2.7 \mu \mathrm{L}$ of $5 \mathrm{M} \mathrm{NaCl}$ was added, followed by incubation for $5 \mathrm{~min}$. After centrifuging at 15,000 rpm for $5 \mathrm{~min}$, the supernatant was removed and $100 \mu \mathrm{L}$ of 4.5\% PEG 6000, $0.5 \mathrm{M} \mathrm{NaCl}, 5 \mathrm{mM}$ Tris- $\mathrm{HCl}(\mathrm{pH} 8.0$ ) was added. After centrifuging again for $1 \mathrm{~min}$, the supernatant was removed and the pellet was dissolved in $10 \mu \mathrm{L}$ of EB. In our experience, centrifugation with salt rather than Ampure beads resulted in a higher library yield and a shorter centrifugation time was preferable. It was extremely rare to find reads containing more than two copies of rDNA with this method, indicating that the in vitro Cas9 efficiency is sufficient. The four gRNA target sequences were 5'ATGAACCGAACGCCGGGTTAAGG, 5'-AGGACGGTGGCCATGGAAGTCGG, 5’ACCTCCACCAGAGTTTCCTCTGG and 5'-TATCCTGAGGGAAACTTCGGAGG.

\section{Mice}

Eight-week-old C57BL6/JJc1 mice were purchased from CLEA Japan, Inc (Tokyo, Japan). Bone marrow cells were extracted as described previously (Madaan et al. 2014). All experiments were approved by the Animal Experiment Ethics Committees of the University of Tokyo (Experiment No. 0210) and performed in accordance with the provided manual.

\section{Cell culture}

EBV-transformed B cells were obtained from National Institutes of Biomedical Innovation, Health and Nutrition. 201B7 hiPSCs were obtained from Riken BioResource Research Center. EBV-transformed B cells were cultured as a floating culture in T25 flasks containing RPMI1640 supplemented with 10\% FBS. hiPSCs were cultured on vitronectin-coated plates with AK02N medium. ht7 mESCs were cultured on $0.1 \%$ gelatincoated plates with standard GMEM-based medium (10\% FCS, 1xNEAA, $1 \mathrm{mM}$ sodium pyruvate. $10^{-4} \mathrm{M} 2-$ ME, $1000 \mathrm{U} / \mathrm{mL} \mathrm{mLIF}$ ). A0031 Werner syndrome patient cells and the iPSCs derived from them were cultured as described previously (Shimamoto et al. 2014). 


\section{Screening of rDNA-derived Oxford Nanopore reads}

553 To analyze the HPGP whole genome sequencing samples, we downloaded the fast5, fastq and sequencing summary files. First, based on the sequencing summary file, we excluded reads that did not pass the sequencing quality filtering. Next, each read was split and mapped to the rDNA reference file (KY962518.1) by using BWA MEM (v0.7.17) and the "ont2d" option. We only used reads that had more than 40,000 nt of continuous rDNA region at either end. Moreover, to remove reads derived from a microsatellite stretch similar to that included in the IGS of rDNA, we checked whether the reads contained at least $10 \%$ of splitreads that mapped to the coding region.

\section{Visualization of the rDNA-derived Oxford Nanopore reads}

562 Each fastq read was split into smaller reads of 300-nt, and mapped to the rDNA reference sequence by using BWA MEM (v0.7.17) as described above. The split-reads were then visualized as lines based on their mean score was plotted. Visualization of CpG methylation was done similarly by binning reads in 200 -nt bins. For each bin, the frequency of methylation was calculated based on the "average" (see below) and the value was plotted as a bar.

\section{Finding non-canonical copies}

570 First, the read was split and mapped to the rDNA reference sequence. If more than $10 \%$ of the mapped 571 segment was in the opposite direction to the dominant direction, the read was classified as inverted and plotted. If the distance between each mapped read differed from the expected length by more than $500 \mathrm{nt}$, the reads were plotted as potential reads containing non-canonical copies. In case that both of two neighboring reads were within the $\mathrm{R}$ repeat or Butterfly/Long repeat region, we did not count them as aberrant copies owing to the natural variation in these regions. Each plotted read was then manually classified based on the visualization. 
Using BWA MEM software, 500-nt rDNA sections located at 10,000, 20,000 and 30,000 nt in the reference sequence were mapped to each read. Next, the distance between the mapped positions of 500-nt sections at 10,000 and 20,000 in each read was used to estimate the $\mathrm{R}$ repeat length and the distance between the mapped positions of 20,000 and 30,000 was used to estimate the Butterfly/Long repeat length. Because genomic mutations in rDNA are rare, most of the variations obtained by this method should be due to repeat length variation in the repeat regions.

\section{Methylation analysis}

587 For the reads that were thought to contain rDNA, fast5 files were extracted by using ont_fast5_api and basecalled by using Guppy Basecaller v4.2.2 with dna_r9.4.1_450bps_modbases_dam-dem-cpg_hac_prom configuration. For threshold-based methylation analysis, we used 0.8 as the threshold for posterior probability. In the HPGP database, there are two types of Nanopore data, which were generated by NHGRIUSCS and HPRC. For the analysis comparing transcribed-region methylation frequency and rDNA copy number, we used only data generated by HPRC because they were submitted to the database over a short period of time and thus were likely to be less affected by differences in experimental conditions. The number of samples available was sufficient for the analysis ( 23 samples).

\section{Whole genome bisulfite sequencing analysis}

597 Fastq files were first cleaned up with Trim Galore! (v0.6.6) to remove adapters (Martin 2011). The frequency 598 of CpG methylation in the rDNA coding region was then estimated by Bismark software (v0.22.3) (Krueger 599 and Andrews 2011) and Bowtie2 (v2.3.5) using the reference genome that contained only the rDNA coding 600 region sequence. We used the following data: m14 mESC (SRR610046, SRA), Rex1 mESC (SRR5099302, 601 SRA), H1 hESC (ENCFF311PSV, ENCODE project) and H9 hESC (ENCFF384QMG, ENCODE project). 602 Files are available through SRA (https://www.ncbi.nlm.nih.gov/sra/) and ENCODE 603 (https://www.encodeproject.org/files/), respectively. 
All of the raw Cas9-enriched data generated in this study have been uploaded to Mendeley Data

\section{COMPETING INTEREST STATEMENT}

611 The authors declare that they have no conflict of interest.

\section{ACKNOWLEDGEMENTS}

614 We thank the members of Kobayashi lab for their useful discussion. This work was supported by AMED-

CREST under grant number JP20gm1110010 to T.K.

\section{REFERENCES}

618 Agrawal S, Ganley ARD. 2018. The conservation landscape of the human ribosomal RNA gene repeats. PLoS One 13: 1-31.

Akamatsu Y, Kobayashi T. 2015. The Human RNA Polymerase I Transcription Terminator Complex Acts as a Replication Fork Barrier That Coordinates the Progress of Replication with rRNA Transcription Activity. Mol Cell Biol 35: 1871-1881.

Burkhalter MD, Sogo JM. 2004. rDNA enhancer affects replication initiation and mitotic recombination: Fob1 mediates nucleolytic processing independently of replication. Mol Cell 15: 409-421.

Caburet S, Conti C, Schurra C, Lebofsky R, Edelstein SJ, Bensimon A. 2005. Human ribosomal RNA gene arrays display a broad range of palindromic structures. Genome Res 15: 1079-1085.

Carrero D, Soria-Valles C, López-Otín C. 2016. Hallmarks of progeroid syndromes: Lessons from mice and reprogrammed cells. DMM Dis Model Mech 9: 719-735.

Cherlin T, Magee R, Jing Y, Pliatsika V, Loher P, Rigoutsos I. 2020. Ribosomal RNA fragmentation into 632 de Lannoy C, Ridder D De, Risse J. 2018. The long reads ahead : de novo genome assembly using the

Defossez P-A, Prusty R, Kaeberlein M, Lin S-J, Ferrigno P, Silver PA, Keil RL, Guarente L. 1999. short RNAs (rRFs) is modulated in a sex- and population of origin-specific manner. BMC Biol 18: 13: 447-455. doi:https://doi.org/10.1016/S1097-2765(00)80472-4. 
Gangloff S, Zou H, Rothstein R. 1996. Gene conversion plays the major role in controlling the stability of large tandem repeats in yeast. EMBO J 15: 1715-1725.

Ganley ARD, Kobayashi T. 2007. Highly efficient concerted evolution in the ribosomal DNA repeats: total rDNA repeat variation revealed by whole-genome shotgun sequence data. Genome Res 17: 184-191.

Ganley ARD, Kobayashi T. 2011. Monitoring the rate and dynamics of concerted evolution in the ribosomal DNA repeats of saccharomyces cerevisiae using experimental evolution. Mol Biol Evol 28: 2883-2891.

Ganley ARD, Kobayashi T. 2014. Ribosomal DNA and cellular senescence: new evidence supporting the connection between rDNA and aging. FEMS Yeast Res 14: 49-59.

Gilpatrick T, Lee I, Graham JE, Raimondeau E, Bowen R, Heron A, Downs B, Sukumar S, Sedlazeck FJ, Timp W. 2020. Targeted nanopore sequencing with Cas9-guided adapter ligation. Nat Biotechnol 38: $433-438$.

Gonzalez IL, Sylvester JE. 2001. Human rDNA: Evolutionary patterns within the genes and tandem arrays derived from multiple chromosomes. Genomics 73: 255-263.

Grozdanov P, Georgiev O, Karagyozov L. 2003. Complete sequence of the 45-kb mouse ribosomal DNA repeat: Analysis of the intergenic spacer. Genomics 82: 637-643.

Grummt I, Rosenbauer H, Niedermeyer I, Maier U, Öhrlein A. 1986. A repeated 18 bp sequence motif in the mouse rDNA spacer mediates binding of a nuclear factor and transcription termination. Cell 45: 837846.

Gupta S, Santoro R. 2020. Regulation and Roles of the Nucleolus in Embryonic Stem Cells: From Ribosome Biogenesis to Genome Organization. Stem Cell Reports 15: 1206-1219.

Kaeberlein M, McVey M, Guarente L. 1999. The SIR2/3/4 complex and SIR2 alone promote longevity in Saccharomyces cerevisiae by two different mechanisms. Genes Dev 13: 2570-2580.

Kass SU, Landsberger N, Wolffe AP. 1997. DNA methylation directs a time-dependent repression of transcription initiation. Curr Biol 7: 157-165.

Killen MW, Stults DM, Adachi N, Hanakahi L, Pierce AJ. 2009. Loss of Bloom syndrome protein destabilizes human gene cluster architecture. Hum Mol Genet 18: 3417-3428.

Kim JH, Dilthey AT, Nagaraja R, Lee HS, Koren S, Dudekula D, Wood WH, Piao Y, Ogurtsov AY, Utani $\mathrm{K}$, et al. 2018. Variation in human chromosome 21 ribosomal RNA genes characterized by TAR cloning and long-read sequencing. Nucleic Acids Res 46: 6712-6725.

Kobayashi T. 2008. A new role of the rDNA and nucleolus in the nucleus - RDNA instability maintains

Kobayashi T. 2011. Regulation of ribosomal RNA gene copy number and its role in modulating genome integrity and evolutionary adaptability in yeast. Cell Mol Life Sci 68: 1395-1403. 
Kobayashi T. 2014. Ribosomal RNA gene repeats, their stability and cellular senescence. Proc Japan Acad Ser B Phys Biol Sci 90: 119-129.

Kobayashi T. 2003. The Replication Fork Barrier Site Forms a Unique Structure with Fob1p and Inhibits the

Kobayashi T, Ganley ARD. 2005. Recombination regulation by transcription-induced cohesin dissociation in rDNA repeats. Science (80-) 309: 1581-1584.

Kobayashi T, Heck DJ, Nomura M, Horiuchi T. 1998a. Expansion and contraction of ribosomal DNA repeats in Saccharomyces cerevisiae: requirement of replication fork blocking (Fob1) protein and the role of RNA polymerase I. Genes Dev 12: 3821-3830.

Kobayashi T, Heck DJ, Nomura M, Horiuchi T. 1998b. Expansion and contraction of ribosomal DNA repeats in Saccharomyces cerevisiae: Requirement of replication fork blocking (Fob1) protein and the role of RNA polymerase I. Genes Dev 12: 3821-3830.

Kobayashi T, Horiuchi T, Tongaonkar P, Vu L, Nomura M. 2004. SIR2 regulates recombination between

Madaan A, Verma R, Singh AT, Jain SK, Jaggi M. 2014. A stepwise procedure for isolation of murine bone

Malinovskaya EM, Ershova ES, Golimbet VE, Porokhovnik LN, Lyapunova NA, Kutsev SI, Veiko NN, Kostyuk S V. 2018. Copy number of human ribosomal genes with aging: Unchanged mean, but narrowed range and decreased variance in elderly group. Front Genet 9: 306.

Martin M. 2011. Cutadapt removes adapter sequences from high-throughput sequencing reads.

Nichols J, Smith A. 2009. Naive and Primed Pluripotent States. Cell Stem Cell 4: 487-492.

Nishino K, Umezawa A. 2016. DNA methylation dynamics in human induced pluripotent stem cells. Hum Cell 29: 97-100.

Niwa H, Miyazaki JI, Smith AG. 2000. Quantitative expression of Oct-3/4 defines differentiation, dedifferentiation or self-renewal of ES cells. Nat Genet 24: 372-376. 
Parks MM, Kurylo CM, Dass RA, Bojmar L, Lyden D, Vincent CT, Blanchard SC. 2018. Variant ribosomal RNA alleles are conserved and exhibit tissue-specific expression. Sci Adv 4: eaao0665.

Petes TD. 1979. Yeast ribosomal DNA genes are located on chromosome XII. Proc Natl Acad Sci U S A 76: $410-4$.

Roussel P, André C, Comai L, Hernandez-Verdun D. 1996. The rDNA transcription machinery is assembled during mitosis in active NORs and absent in inactive NORs. $J$ Cell Biol 133: 235-246.

Saka K, Ide S, Ganley ARD, Kobayashi T. 2013. Cellular senescence in yeast is regulated by rDNA

Santoro R, Grummt I. 2005. Epigenetic Mechanism of rRNA Gene Silencing: Temporal Order of NoRCMediated Histone Modification, Chromatin Remodeling, and DNA Methylation. Mol Cell Biol 25: 2539-2546.

Schawalder J, Paric E, Neff NF. 2003. Telomere and ribosomal DNA repeats are chromosomal targets of the bloom syndrome DNA helicase. BMC Cell Biol 4.

Shafin K, Pesout T, Lorig-Roach R, Haukness M, Olsen HE, Bosworth C, Armstrong J, Tigyi K, Maurer N, Koren S, et al. 2020. Nanopore sequencing and the Shasta toolkit enable efficient de novo assembly of eleven human genomes. Nat Biotechnol 38: 1044-1053.

Shimamoto A, Kagawa H, Zensho K, Sera Y, Kazuki Y, Osaki M, Oshimura M, Ishigaki Y, Hamasaki K, Kodama Y, et al. 2014. Reprogramming suppresses premature senescence phenotypes of Werner syndrome cells and maintains chromosomal stability over long-term culture. PLoS One 9: 1-13.

Sinclair DA, Guarente L. 1997. Extrachromosomal rDNA Circles-A Cause of Aging in Yeast. Cell 91:

Takeuchi Y, Horiuchi T, Kobayashi T. 2003. Transcription-dependent recombination and the role of fork collision in yeast rDNA. Genes Dev 17: 1497-1506.

Tomoda K, Takahashi K, Leung K, Okada A, Narita M, Yamada NA, Eilertson KE, Tsang P, Baba S, White MP, et al. 2012. Derivation conditions impact X-inactivation status in female human induced pluripotent stem cells. Cell Stem Cell 11: 91-9.

van Sluis M, van Vuuren C, Mangan H, McStay B. 2020. NORs on human acrocentric chromosome p-arms

Wang M, Lemos B. 2019. Ribosomal DNA harbors an evolutionarily conserved clock of biological aging. Genome Res 29: 325-333. DNA Variations in Mice. Mol Cell Biol 40. 
737 Weitao T, Budd M, Hoopes LLM, Campbell JL. 2003. Dna2 helicase/nuclease causes replicative fork stalling and double-strand breaks in the ribosomal DNA of Saccharomyces cerevisiae. $J$ Biol Chem 278: $22513-22522$.

740 Woolnough JL, Atwood BL, Liu Z, Zhao R, Giles KE. 2016. The regulation of rRNA gene transcription during directed differentiation of human embryonic stem cells. PLoS One 11: 1-18. 